\title{
Identification of the neurotransmitter profile of AmFoxP expressing neurons in the honeybee brain using double-label in situ hybridization
}

\author{
Adriana Schatton ${ }^{1 *} \mathbb{0}$, Julia Agoro ${ }^{1,2}$, Janis Mardink' ${ }^{1}$ Gérard Leboulle ${ }^{2}$ and Constance Scharff ${ }^{1}$
}

\begin{abstract}
Background: FoxP transcription factors play crucial roles for the development and function of vertebrate brains. In humans the neurally expressed FOXPs, FOXP1, FOXP2, and FOXP4 are implicated in cognition, including language. Neural FoxP expression is specific to particular brain regions but FoxP1, FoxP2 and FoxP4 are not limited to a particular neuron or neurotransmitter type. Motor- or sensory activity can regulate FoxP2 expression, e.g. in the striatal nucleus Area $X$ of songbirds and in the auditory thalamus of mice. The DNA-binding domain of FoxP proteins is highly conserved within metazoa, raising the possibility that cellular functions were preserved across deep evolutionary time. We have previously shown in bee brains that FoxP is expressed in eleven specific neuron populations, seven tightly packed clusters and four loosely arranged groups.
\end{abstract}

Results: The present study examined the co-expression of honeybee FoxP (AmFoxP) with markers for glutamatergic, GABAergic, cholinergic and monoaminergic transmission. We found that AmFoxP could co-occur with any one of those markers. Interestingly, AmFoxP clusters and AmFoxP groups differed with respect to homogeneity of marker co-expression; within a cluster, all neurons co-expressed the same neurotransmitter marker, within a group co-expression varied. We also assessed qualitatively whether age or housing conditions providing different sensory and motor experiences affected the AmFoxP neuron populations, but found no differences.

Conclusions: Based on the neurotransmitter homogeneity we conclude that AmFoxP neurons within the clusters might have a common projection and function whereas the AmFoxP groups are more diverse and could be further sub-divided. The obtained information about the neurotransmitters co-expressed in the AmFoxP neuron populations facilitated the search of similar neurons described in the literature. These comparisons revealed e.g. a possible function of AmFoxP neurons in the central complex. Our findings provide opportunities to focus future functional studies on invertebrate FoxP expressing neurons. In a broader context, our data will contribute to the ongoing efforts to discern in which cases relationships between molecular and phenotypic signatures are linked evolutionary.

Keywords: FoxP, FoxP1, Honeybee, Acetylcholine, Glutamate, GABA, Monoamine, Songbird, Deep homology, In situ hybridization

\footnotetext{
*Correspondence: a.schatton@fu-berlin.de

${ }^{1}$ Department of Animal Behavior, Freie Universität Berlin, Takustraße 6,

14195 Berlin, Germany

Full list of author information is available at the end of the article
}

(c) The Author(s) 2018. This article is distributed under the terms of the Creative Commons Attribution 4.0 International License (http://creativecommons.org/licenses/by/4.0/), which permits unrestricted use, distribution, and reproduction in any medium, provided you give appropriate credit to the original author(s) and the source, provide a link to the Creative Commons license, and indicate if changes were made. The Creative Commons Public Domain Dedication waiver (http://creativecommons.org/ publicdomain/zero/1.0/) applies to the data made available in this article, unless otherwise stated. 


\section{Background}

Transcription factors of the FOXP family are intensively investigated because of their role in human disease [1]. Human patients with mutations in FOXP1, FOXP2 or FOXP4 show several cognitive deficits such as autistic features, mental retardation and language impairments [2-6]. A point mutation in FOXP2, initially discovered in one large family, impairs speech perception and production while non-language-related behaviors are less affected [7, 8]. More than 30 genetic alterations of FOXP2 in other individuals also affect language in similar ways, and are additionally associated with autistic spectrum disorders $[5,9-11]$. In the following text we adopted the nomenclature proposed by Kaestner et al. [12], e.g. human gene FOXP and protein FOXP, mouse (Foxp2/Foxp2) and all other species (FoxP2/FoxP2).

FoxP gene expression in the CNS is overall similar in all vertebrates analyzed and includes regions involved in sensory-motor and multimodal integration [13-23].

Invertebrate genomes harbor only one FoxP gene locus that contains a highly conserved DNA-binding domain $[24,25]$. In sponges FoxP is upregulated in vitro during the formation of cell aggregates [26]. In Drosophila, FoxP (dFoxP) has been implicated in decision-making, locomotion and motor learning [27-30]. In honeybees, FoxP (AmFoxP) is expressed in the cortical areas of all major neuropils, either in dense neuron clusters or more widely dispersed neuron groups [25, 31,32]. The FoxP gene is most similar to vertebrate FoxP1, based on particular alternative splice forms in bilaterians [25, 33].

We previously described eleven AmFoxP expressing neuron populations in particular locations of the honeybee brain [25]. We chose to study the role of FoxP in the honeybee Apis mellifera because it is a well-established animal model to study insect cognition $[34,35]$ such as memory formation [36], navigation [37], symbolic communication [38-41] or conceptual learning [42-44].

For one of the described AmFoxP clusters, the posterior cluster medioventral to the lobula (mvLO-p), we proposed, based on location and connectivity, a functional role in visual processing $[25,31]$, consistent with FoxP expressing neurons in the mammalian visual thalamus $[45,46]$. Here we further characterize the AmFoxP neuron populations in the honeybee brain in terms of their neurotransmitters to better compare them with neurons of known function in the insect CNS. Moreover, we also studied whether environmental stimuli affected AmFoxP expression in honeybees, because FoxP2 can be regulated by activity in some vertebrate neurons, e.g. by light, mtor behavior and/or sound [13, 47-49].

In honeybees, neurotransmitter systems were described by various means, including the detection of the neurotransmitters themselves [50-55], of the enzymes implicated in their metabolism [56-58], of molecules that transport the neurotransmitters into cellular compartments [59] and of specific receptors [60-67].

We concentrated on the most relevant neurotransmitters by means of their markers: the honeybee (Am) vesicular transporters for acetylcholine (AmVAchT), glutamate (AmVGluT) and monoamines (AmVMAT) as well as glutamate decarboxylase (AmGad). These markers were chosen because they are exclusively associated with presynaptic release (transporters) or synthesis (Gad) of the respective neurotransmitter. To our knowledge, VAchT, VGluT and Gad have not been reported to be expressed endogenously in glia cells. Drosophila VMAT is also expressed in glia cells, however restricted to serotonin and histamine containing neurons in a relatively thin layer between the retina and the optic lobe [68] where we did not detect AmFoxP. Acetylcholine (Ach) is the main excitatory neurotransmitter in insects, in contrast to vertebrates that predominantly use glutamate for excitatory transmission [69, 70]. Excitatory currents induced by Ach are characterized [71, 72]. Glutamatergic signaling in honeybees is important for particular memory mechanisms $[63,73,74]$. Inhibitory currents induced by Glutamate (Glu) through glutamate chloride channels (GluCl) have also been described [72, 75]. The detection of receptors homologous to their vertebrate counterparts (AMPA/kainate, NMDA) suggests that Glu also acts as an excitatory neurotransmitter in the CNS [61]. Another major inhibitory neurotransmitter in the bilaterian brain is GABA [72, 76, 77]. GluCl and GABA receptors are both involved in honeybee long term memory $[78,79]$. AmVMAT transports the biogenic amines octopamin, dopamine, histamine and serotonin $[68,80]$ and thus serves as a marker for modulatory neurons. In insects, modulatory neurons are crucial for different learning processes [81-83], involved in the division of labour [84, $85]$ and mediate the biological state of the organism like aggression, sleep or hunger [86-88]. For the quantitative PCR analysis of neurotrasmitters expressed in the Kenyon cells of the mushroom bodies, we used, beside AmVachT, AmVGluT and AmVMAT, also AmVIAAT (the honeybee vesicular transporter for inhibitory amino acids). AmVIAAT is a presynaptic marker for glycinergic and GABAergic neurons.

Although insect and vertebrate brains demonstrate fundamental differences in terms of development, anatomy, structure, design and complexity, they also have many principle features in common [89]. Some of those might be convergent whereas others, like the regulatory networks and molecular architecture of specific neurons could date back to their last common bilaterian ancestor and are therefore 'deeply homologous' [90]. In the framework of deep homology between insect and vertebrate 
brains [90, 91], we are interested in potentially conserved features of FoxP expressing neurons with implications for similarities and differences in behavior.

\section{Methods}

\section{Animals}

All honeybees of the species A. mellifera were collected between July and September from apiaries located at the department of Neurobiology, FU Berlin (Königin-LuiseStr. 1-3, 14195 Berlin). Access was generously provided by Professors Randolf Menzel and Dorothea Eisenhardt.

\section{Sampling method}

To address whether differences in experience or life history affected AmFoxP expression we created four conditions that varied with respect to sensory experience and opportunity for motor activity. From each condition we sampled bees of different ages. In condition 1 bees were kept in an observation hive with two frames $(\mathrm{W} \times \mathrm{D} \times \mathrm{H}: 53.5 \times 18 \times 65 \mathrm{~cm})$, in the presence of the queen and siblings ( 4000 individuals), a honeycomb and the possibility to fly and forage outside of the hive ('unmanipulated' group). In order to sample bees of the unmanipulated group at specific ages, they were marked with acrylic color on their thorax within $16 \mathrm{~h}$ after emergence. In the other three conditions one or more variables were altered (Table 1): In condition 2 ('honeycomb' group) 100 individuals were kept in a caged honeycomb frame $(22 \mathrm{~cm} \times 37 \mathrm{~cm} \times 5 \mathrm{~cm}$ ). In condition 3 ('minicage' group) $\sim 30$ bees were kept separately within the hive, enclosed in a small cage $(10 \mathrm{~cm} \times 3 \mathrm{~cm} \times 10 \mathrm{~cm})$ without an own honeycomb. In condition 4 ('incubator' group) $\sim 30$ bees were kept in an empty cage $(13 \mathrm{~cm} \times 20 \mathrm{~cm} \times 8 \mathrm{~cm})$ within an incubator at $29^{\circ} \mathrm{C}$ in constant darkness and supplied with $30 \%$ sucrose.

\section{Preparation of in situ hybridization probes}

Table 2 provides an overview of the primers used to prepare in situ hybridization (ISH) probes. In the following text, nucleotide sequences (ISH-probes, primers) are written in italics and 'Am' as a prefix refers to 'Apis mellifera'. The AmFoxP probe-containing pGemT-easy plasmid was kindly provided by Prof. Taketoshi Kiya (Kanazawa University, Japan). The same probe was used

Table 1 Treatment and sample size of the four experimental groups

\begin{tabular}{lllll}
\hline Condition/group & Sisters & Queen & Honeycomb
\end{tabular}

The manipulated features are indicated by symbols. The column on the right lists the sample size. The detailed distribution of age ( $d=$ days after emergence) within the group is given in brackets

Table 2 RNA probe names used for in situ hybridization

\begin{tabular}{|c|c|c|c|}
\hline Probe name & Primers $5^{\prime}-3^{\prime}$ & Ref. seq of cds & Length (nt) \\
\hline \multirow[t]{2}{*}{ AmFoxP } & GAGAAACCGCTGGACGTTTC & NM_001104949 & 847 \\
\hline & CGTTGCGCCGGAAGTAGCAG & & \\
\hline \multirow[t]{2}{*}{ AmVGlut } & GGCCCCCATTGCGTCACA & XM_016914051.1 & 631 \\
\hline & AATGCCAGCCACAACCAGAAACAGTA & & \\
\hline \multirow[t]{2}{*}{ AmGad } & AATGGTGAACGTCTGCTTCTGGTAT & XM_391979 & 806 \\
\hline & ACTTACGTGCTATGAGTATCCTTTG & & \\
\hline \multirow[t]{2}{*}{ AmVAchT } & CTCGGGCGCGTTGATAGACAGGAT & XM_006562557.2 & 605 \\
\hline & CGCCGAACACGTGGGGGAAGAA & & \\
\hline \multirow[t]{2}{*}{ AmVMAT } & AAGGCGTTGGTTCGTCGTGCTC & XM_392061.6 & 855 \\
\hline & CTTCTTTCGTTGGCGGTGCTCGTAA & & \\
\hline \multirow[t]{2}{*}{ D2-like/AmDop3 } & CAGCGCATTCGTTAATCTGA & NM_001014983.1 & 534 \\
\hline & GCCCAGACCAACAGTATCGT & & \\
\hline
\end{tabular}


previously [25] and does not discriminate between the two the Forkhead domain-affecting isoforms [25]. The AmGad-probe was produced according to Kiya and Kubo [58]. Honeybee sequences were identified with BLASTN 2.7.0+ using the 'blastn' algorithm [92] (RRID: 196SCR_001010) and phylogenetic analysis (clustal omega, EMBL-EBI, RRID:SCR_001591) by comparing to Drosophila and mammalian orthologous genes available in sequence databases. Primers (mwg Eurofins Genomics, Ebersberg, Germany) were identified with 'primer3' software [93] (RRID:SCR_002285) and used to PCRamplify sequences between 500 and 1000 nt to serve as ISH templates. Template cDNA was prepared as follows: freshly dissected honeybee brains were homogenized with a pellet pestle (Sigma-Aldrich, Germany) in $400 \mu \mathrm{l}$ TriZol $^{\circledR}$. After adding $80 \mu \mathrm{l}$ of chloroform the solution was vortexed and centrifuged $\left(15 \mathrm{~min}\right.$ at $12,000 \mathrm{~g}$ at $4{ }^{\circ} \mathrm{C}$ ), the RNA-containing upper layer was collected and purified on columns ('RNeasy MinElute Cleanup' by Qiagen, Hilden, Germany) according to the manufacturer's protocol. Residual DNA was restricted with TurboDNase ${ }^{\circledR}$ (Thermo Fisher, Braunschweig, Germany). RNA concentration was measured with a NanoDrop 1000 Spectrophotometer. $200 \mathrm{ng}$ of total RNA were transcribed into cDNA by using oligo-dT-primers and SuperScript ${ }^{\circledR}$ III Reverse Transcriptase (Thermo Fisher, Braunschweig, Germany).

PCR-amplified fragments were purified with a purification kit (Macherey-Nagel, Düren, Germany) and ligated into pGemT-easy plasmids (Promega, Wisconsin, USA) which were transformed in E. coli bacteria (Top 10). DNA-templates for RNA-probe synthesis were PCRamplified with pGemt-easy M13 primers, detected by gel electrophoresis and purified again. SP6 and T7 polymerases (Roche, Mannheim, Germany) were used for in vitro transcription with Dig- and FITC-labeled UTP containing RNA Labeling Mix (Roche, Mannheim, Germany) and $200 \mathrm{ng}$ of the cDNA template. Probes were purified with mini Quick Spin Columns (Roche, Mannheim, Germany), diluted $1: 1$ in formamid and stored at $-80^{\circ} \mathrm{C}$.

\section{Tissue preparation}

Honeybee brains were quickly dissected in DEPC-treated water, immediately embedded in TissueTek ${ }^{\circledR}$ (Sakura, Staufen, Germany), frozen on dry ice and kept at $-80{ }^{\circ} \mathrm{C}$ until use.

\section{Staining protocol-fluorescent double label}

The double-label ISH (dISH) protocol was kindly provided by Prof. T. Kiya (Kanazawa University), published in Kiya and Kubo [58] and was slightly modified. Briefly, $12 \mu \mathrm{m}$ frontal cryosections were fixed $15 \mathrm{~min}$ in $4 \%$ PFA in DEPC-treated water at room temperature (RT), washed in $0.1 \mathrm{M}$ phosphate buffer $\mathrm{pH}$ 7.4 (PB: $18 \mathrm{mM}$ $\mathrm{NaH}_{2} \mathrm{PO}_{4}, 82 \mathrm{mM} \mathrm{Na}_{2} \mathrm{HPO}_{4}$ ), permeabilized and covered with the respective Dig- and FITC-labeled RNA probes. Per slide $0.3 \mu \mathrm{l}$ AmFoxP and $1 \mu \mathrm{l}$ neurotransmission marker probes were diluted in $150 \mu \mathrm{l}$ hybridization buffer [58]. Slides were covered with coverslips $(24 \times 60 \mathrm{~mm}$, Carl Roth, Karlsruhe, Germany) and hybridized overnight at $60{ }^{\circ} \mathrm{C}$ in a mineral oil bath. The next day, slides were washed sequentially in $5 \times, 2 \times$ and $0.2 \times$ salinesodium citrate buffer pH $7.0(20 \times$ SSC: $3 \mathrm{M} \mathrm{NaCl}$, $0.3 \mathrm{M}$ trisodium citrate), blocked in blocking reagent (Roche, Mannheim, Germany) and incubated $2 \mathrm{~h}$ at RT with anti-Dig-POD antibody (1:500, Roche, Mannheim, Germany). RNAse A treatment was not applied. After several washes, slides were incubated $15 \mathrm{~min}$ at RT in Cy5-labled tyramids ('TSA-system' by Perkin Elmer, Rodgau, Germany) according to the manufacturer's protocol. Peroxidase was reduced by $3 \%$ hydrogen peroxide, the slides blocked again and incubated overnight at $4{ }^{\circ} \mathrm{C}$ with anti-FITC-POD (1:500, Roche, Mannheim, Germany). The next day, slides were washed and incubated in Cy3labled tyramids ('TSA-system' by Perkin Elmer, Rodgau, Germany), counterstained with DAPI for $10 \mathrm{~min}$ at RT $(1: 20,000)$ and embedded in 'Immu-Mount ${ }^{\mathrm{TM},}$ (Thermo Fisher, Braunschweig, Germany).

Adjacent cryosections were incubated each with the AmFoxP-specific Dig-labeled probe and a second FITC-labeled probe specific for one of the four neurotransmitter markers, which were the honeybee $(A m)$ vesicular transporters of either (1) acetylcholine (AmVAchT), (2) glutamate (AmVGluT) and (3) monoamines (AmVMAT) or (4) glutamate decarboxylase $($ AmGad) (Tables 2, 4).

We also performed one $d I S H$ with the FITC-labeled AmVAchT and a Dig-labeled AmVGluT probe on a newly emerged bee. Sample sizes for bees analyzed for neurotransmitter markers are listed in Table 1 . For the analysis of the monoaminergic receptor AmDop3 in the $\mathrm{KC}$, one unmanipulated adult forager of undetermined age was analyzed using $d I S H$.

Probes were chosen for $d I S H$ if two conditions were met: for each probe, the corresponding control ('sense') probe produced no specific staining and the 'antisense' probe labeled neurons known to express the particular neurotransmitter, e.g.: the octopaminergic VUMmx neurons located ventrally to the gnathal ganglia (GNG) for AmVMAT [53], the glutamatergic cortex of the optic lobes for AmVGluT [52], the inhibitory local interneurons and inhibitory projection neurons lateral and dorsal to the antennal lobes (AL) $[50,57,94]$ as well as the neurons of the protocerebral-calycal tract (p.c.t.) $[95,96]$ for $A m G a d$ and the uniglomerular projection neurons (uPNs) of the antennal lobes [57, 97, 98] for AmVAchT. 


\section{Chromogen single label}

Tissue was dissected as described for $d I S H$ above but incubated with biotinylated (instead of fluorescently labeled) tyramids and $\mathrm{DAB}$ was used as the chromogen according to the manufacturer's protocol (DAKO/agilent, Santa Clara, USA). All sense and antisense probes were first used in a single-label protocol to check for specificity.

\section{Immuno-labeling of AmFoxP protein}

AmFoxP protein was detected using the custom-made AmFoxP ${ }^{42 k D a}$ antiserum (RRID: AB_2722599). Antiserum specificity was shown previously [25, 32], immunolabeling protocol, microscopy and data analysis was performed as described previously $[25,32]$. Figure $7 \mathrm{~b}$ was prepared from an immuno-labeled brain that was previously injected (in vivo) with Lucifer yellow (Life Technologies L453, CH lithium salt, MW 457.24; 5\%) as described in [25].

\section{RT-qPCR and statistics}

Animals were captured from an observation hive composed of marked bee as described above ('unmanipulated' group, see "Sampling method" section). They were immobilized on ice and decapitated. The head capsule was fixed on wax, the brain was exposed and covered with $0.1 \mathrm{M}$ PBS (137 mM NaCl, $2.7 \mathrm{mM} \mathrm{KCl}, 8.1 \mathrm{mM}$ $\left.\mathrm{Na}_{2} \mathrm{HPO}_{4}\left(2 \mathrm{H}_{2} \mathrm{O}\right), 1.47 \mathrm{mM} \mathrm{KH} \mathrm{PO}_{4}\right)$, the calyces were visually identified, removed with forceps and homogenized with a Teflon pestle and a glass homogenizer filled with $200 \mu \mathrm{l}$ Trizol (Braunschweig, Germany). One sample consisted of 8-10 animals. The precision of the dissection was evaluated by staining brains with SYTOX Green diluted 1:2000 to reveal cell bodies (Fig. 14k, l). Total RNA was extracted and cDNA was synthetized from $1 \mu \mathrm{g}$ of total RNA (see "Preparation of in situ hybridization probes" section). For the RT-qPCR experiments, $5 \mu \mathrm{l}$ of diluted cDNA, $1 \mu \mathrm{l}$ of the forward and reverse primers (10 $\mu \mathrm{M}$, TIB Molbiol, Berlin, Germany), $10 \mu \mathrm{l} \mathrm{Kapa}$ Sybr Fast qPCR mastermix (PeqLab, Erlangen, Germany), $0.4 \mu$ low Rox (50 nM) adjusted to $25 \mu$ with water and analyzed on a Stratagene MX3000P (Agilent Technologies, Santa Clara, USA). Primers are listed in Table 3, AmViaat is the vesicular transporter for inhibitory amino-acids that is specific in vertebrates for GABA and glycine. This candidate was selected instead of AmGad because its expression levels can be better compared to the other vesicular transporters. AmRpL32 and $A m G a p D H$ were chosen as reference genes. Primer efficiency was calculated on serial dilutions $\left(10,10^{2}, 10^{3}\right.$ and $10^{4}$ ) of the cDNA, primer interactions or the formation of unspecific products evaluated by melting curve analysis at the end of the amplification. On each plate, 'no template controls' (NTC) were analyzed by replacing cDNA with water for each parameter. Each sample was analyzed in triplicate. In Fig. 14l, the PCR profile was $2 \min 95^{\circ} \mathrm{C}$, 40 amplification cycles $30 \mathrm{~s}$ at $95^{\circ} \mathrm{C}, 30 \mathrm{~s}$ at $59^{\circ} \mathrm{C}, 30 \mathrm{~s}$ at $72{ }^{\circ} \mathrm{C}$ and the melting curve analysis: $1 \mathrm{~min} 95^{\circ} \mathrm{C}, 30 \mathrm{~s}$ at $55^{\circ} \mathrm{C}$, increasing to $95{ }^{\circ} \mathrm{C}$ and $30 \mathrm{~s}$ at $95^{\circ} \mathrm{C}$. The Cts were calculated by amplification based threshold. Fold change was calculated with the corrected amplification rate: $\mathrm{e}^{-\mathrm{Ct}}$ $\left(\mathrm{e}=10^{-1 / \text { slope }}\right)$ and the data were normalized to the geometric mean of the housekeeper genes, which did not vary over age groups (Kruskal-Walli statistic $\mathrm{H}(2)=0.6$, $p=0.74$ ). Plots and statistics were performed with GraphPad Prism version 5.00 for Windows (GraphPad Software, La Jolla California USA) (RRID: SCR_015807). Data was tested for normal distribution using D'Agostino \& Pearson omnibus normality test. Two means were compared for statistical differences using two-tailed Student's t test.

\section{Results}

AmFoxP expressing neuron populations (clusters and groups)

We determined which of the four investigated neurotransmitter markers, AmVAchT, AmVGLuT, AmVMAT and $A m G a d$, were co-expressed in the 11 AmFoxP neuron populations. We identified these population previously based on mRNA in situ hybridization and immunoreactivity to a custom-made antiserum ('antiAmFoxP $\left.{ }^{42 \mathrm{kDa}^{\prime}}\right)[25,32]$. The anatomical nomenclature

Table 3 RT-qPCR primer list and amplification efficiency

\begin{tabular}{lllc}
\hline $\mathbf{m R N A}$ & Forward $\mathbf{5}^{\prime} \mathbf{3}^{\prime}$ & Reverse $\mathbf{5}^{\mathbf{\prime}} \mathbf{3}^{\mathbf{\prime}}$ & Efficiency (\%) \\
\hline AmVAChT & AGGGCGTCGGTTCCGCTTTC & CAGCATCACTCCGTCCGCCA & 89.7 \\
AmVG/UT & AACGCCCCGTGAGGGTAGCA & GACGCAATGGGGGCCGTTCA & 96.3 \\
AmVMAT & ATTGTCGGCCCCCTCACCCA & GAGCACGACGAACCAACGCC & 99.7 \\
AmVIAAT & CGCCGTATTGCGAGGCGGTT & CGCGTTGTCCAGTCGTCGTGT & 99.8 \\
AmRpL32 & TGTGCTGAAATTGCTCATGGGGG & AGAACGTAACCTTGCACTGGCATAA & 100.3 \\
AmGapDH & CGGTTTTGGCCGTATTGGCCGT & AATGGCAACAACCTGAGCACCGAA & 99.4 \\
\hline
\end{tabular}


follows Ito et al. [99], Table 5. For ease of reading, we will use the terms AmFoxP neuron, AmFoxP cluster and AmFoxP group. We previously showed these cells to produce both AmFoxP mRNA and AmFoxP protein $[25,32]$. Here we use immunohistochemistry and in situ hybridization (ISH) interchangeably. Neurons that were neither immunoreactive to the AmFoxP ${ }^{42 \mathrm{kDa}}$ antiserum nor labeled by the AmFoxP ISH probe are referred to as AmFoxP-negative.

We previously described seven AmFoxP 'clusters', which consist of densely packed neurons and four 'groups', in which the AmFoxP neurons are more broadly distributed ([25], Fig. 1, Table 3 ). In the present study, we found that each of the seven AmFoxP clusters homogeneously co-expressed only one of the used neurotransmitter markers. In contrast, all AmFoxP groups co-expressed two or even three of the transmitter markers, but not AmVMAT. This difference between clusters and groups was observed in all treatment groups and ages.

\section{Neurotransmitter expression}

All four neurotransmitter markers used in the present study were detected in cortical areas around all major neuropils (Figs. 2, 3, 4, 5, 6, 7, 8, 9, 10, 11, 12, 13).

The mRNA of AmVAchT and AmVGluT as well as $A m G a d$ were very abundant in cortical areas surrounding the antennal (AL) (Fig. 3) and optic lobes (OL) (Fig. 4), as well as the gnathal ganglia (GNG) (Fig. 7). The markers of the two excitatory neurotransmitters AmVAchT and AmVGluT had similar expression patterns. However double labeling showed that both AmVAchT and AmVGluT mRNAs were not co-expressed in the same neurons (Fig. 2).

AmVMAT expression was scarce and found in cell clusters (Figs. 3d, 5d) as well as in single cells (Figs. 4d, 7d, l, $8 d, h, 10 e, i, k, 11)$.

\section{Cholinergic AmFoxP neurons}

$A m V A c h T$ was detected in large cell clusters dorsal and dorso-lateral to the AL (Fig. 3c)-most likely
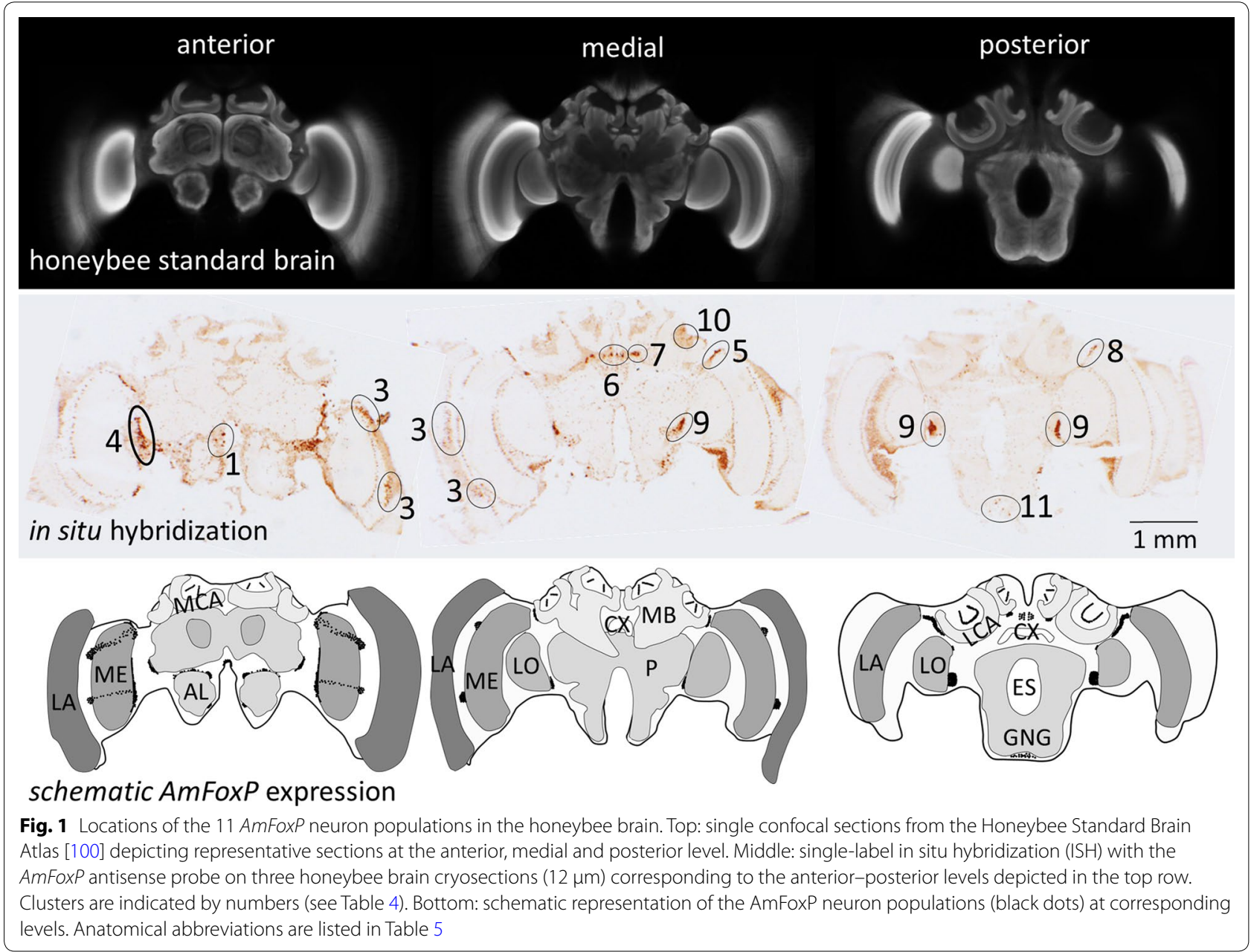

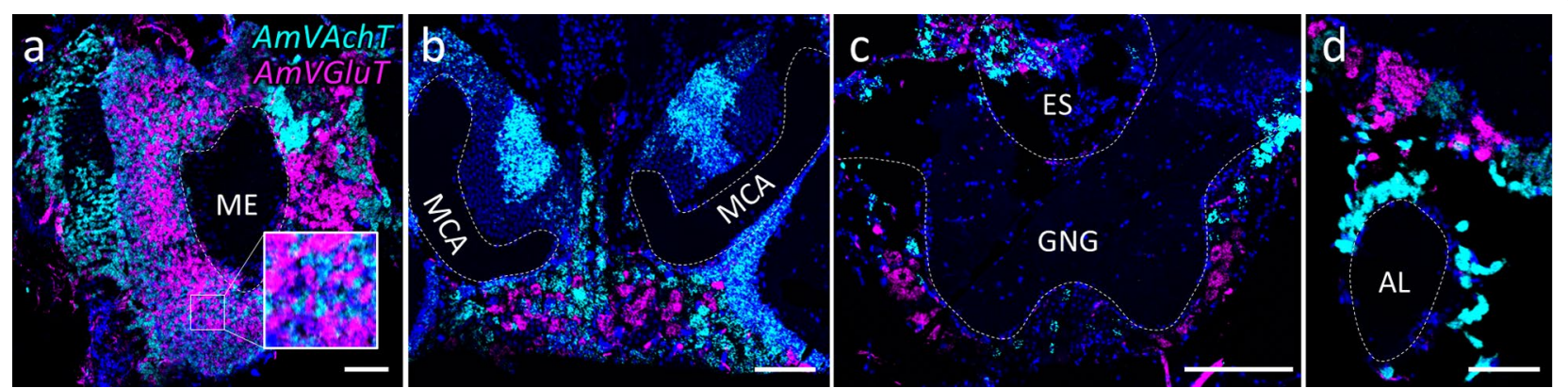

Fig. 2 AmVAchT (cyan) and AmVGluT (magenta) do not co-localize within the same neurons. Double ISH (dISH) stainings on $12 \mu \mathrm{m}$ cryosections from the brain of a newly emerged worker honeybee depict somatic areas surrounding different neuropils: $\mathbf{a}$ the area around the medulla (ME), $\mathbf{b}$ the area ventral to the medial calices (MCA) in the posterior brain, $\mathbf{c}$ the area ventral and lateral to the gnathal ganglia (GNG) and $\mathbf{d}$ the area around the antennal lobes (AL). Rectangular inset in a shows higher magnifications of small boxed region. Scale bars: $100 \mu \mathrm{m}$

uniglomerular projection neurons (uPNs) [57], in cortical areas around the OL and in the KC (Fig. 14a-e). The cholinergic marker was co-expressed in five of the seven AmFoxP clusters (Table 4), i.e. the ILH (Fig. 5), the plLCA (Fig. 6), the vMCA (Fig. 8), the large mvLO (Fig. 10) and the mKC (Fig. 12b-e). The mvLO cluster has a higher cell density than the surrounding tissue, clearly visible in nuclear DAPI staining (Fig. 10i, k). However, by AmFoxP labeling, this cluster could be further subdivided in two subdivisions, an anterior (mvLO-a) and a posterior (mvLO-p) part; each subdivision projects to a different brain area, as described previously [25]. Both subdivisions expressed AmVAchT. The mvLO-a and mvLO-p are separated by a layer of AmFoxP-negative neurons that expressed AmGad (Fig. 10f). AmVAchT was also clearly expressed in the region of the $\mathrm{mKC}$ and coincided with $A m F o x P$ expression. However, due to the subcellular punctate staining in the $\mathrm{KC}$ (Fig. 12bIII-V), the two colors representing AmFoxP and AmVAchT did not coincide to the same extent as in other neurons. We therefore considered $\mathrm{KC}$ to co-express two probes as long as any punctate label was located within the area of a DAPIpositive nucleus, which was the case for AmVAchT in essentially all $A m F o x P$-expressing $\mathrm{KC}$ (but different in the AmDop3/AmFoxP dISH, Fig. 12g). In the AmFoxP groups, with less densely clustered cells and co-expression of more than one neurotransmitter, AmVAchT was expressed in neurons around the AL (pAL, Fig. 3c, j), around the medulla (pMe, Fig. 4c, g, j) and ventral to the GNG (vGNG, Fig. 7c, g) but not dorsal to the central complex (dCX, Fig. 8). The pME could be further subdivided in at least three local groups. Two of them wrap around the anterior medulla; one ventrally and one dorsally (Fig. 4a-g). The ventral AmFoxP neurons co-expressed only AmVAchT (Fig. 4c, g), whereas the dorsal one co-expressed AmVAchT, AmVGluT and
AmGad (Fig. 4c, e-g) and the group lateral to the ME co-expressed AmGad and AmVGluT (Fig. 4e, f, I, k, l).

\section{GABA-ergic AmFoxP neurons}

Our stainings confirmed the AmGad expression pattern shown by Kiya and Kubo [58]. We also found GABAergic neurons particularly in the cortical regions of the $A L$, the $\mathrm{OL}$, the $\mathrm{CX}$, but not in the $\mathrm{KC}$ of the $\mathrm{MB}$, which is also corroborated by our RT-qPCR data (Fig. 14m). AmGad was detected in large clusters lateral to the AL (Fig. 3g, h, o), most likely inhibitory PNs (iPNs) $[94,101]$ or inhibitory local interneurons $[50,57]$. The A3-v neuron cluster of the PCT $[58,95,96]$ was also labeled (not shown). Only one AmFoxP cluster expressed AmGad exclusively, the alLCA (Fig. 6). AmGad expressing AmFoxP neurons were also observed in all four AmFoxP groups; around the AL (pAL, Fig. 3e, g, h, l, o), around the ME (pME, Fig. 4e, I, k), ventral to the GNG (vGNG, Fig. 7e, h, j) and dorsal to the CX (dCX, Fig. 8e, k, i). Within the pME, GABAergic AmFoxP neurons accumulated medioventral to the ME (Fig. 4e, i, k).

\section{Glutamatergic AmFoxP neurons}

AmVGluT was strongly expressed in large somata lateral to the GNG (Fig. 2c), most likely motor neurons as described previously [52], as well as in cortical areas around the medulla (Fig. 2a, Fig. 4f, l) and in clusters dorsal to the AL (Fig. 3f, n, m). Within the pAL glutamatergic AmFoxP neurons were detected anterio-mediodorsal (Fig. 3n) and posterioventral to the AL (Fig. 3i). In seven samples (Fig. 14f-j) there was also very weak AmVGluT signal in the small and middle $K C$ (sKC and mKC) of the MB. In the other eight AmVGluT samples the KC signal did not differ from background. The adES cluster was the only AmFoxP cluster that exclusively co-expressed AmVGlut (Fig. 3f, m). Only few AmVGluT neurons were detected in the vGNG (Fig. 7f) and dorsal to the central 

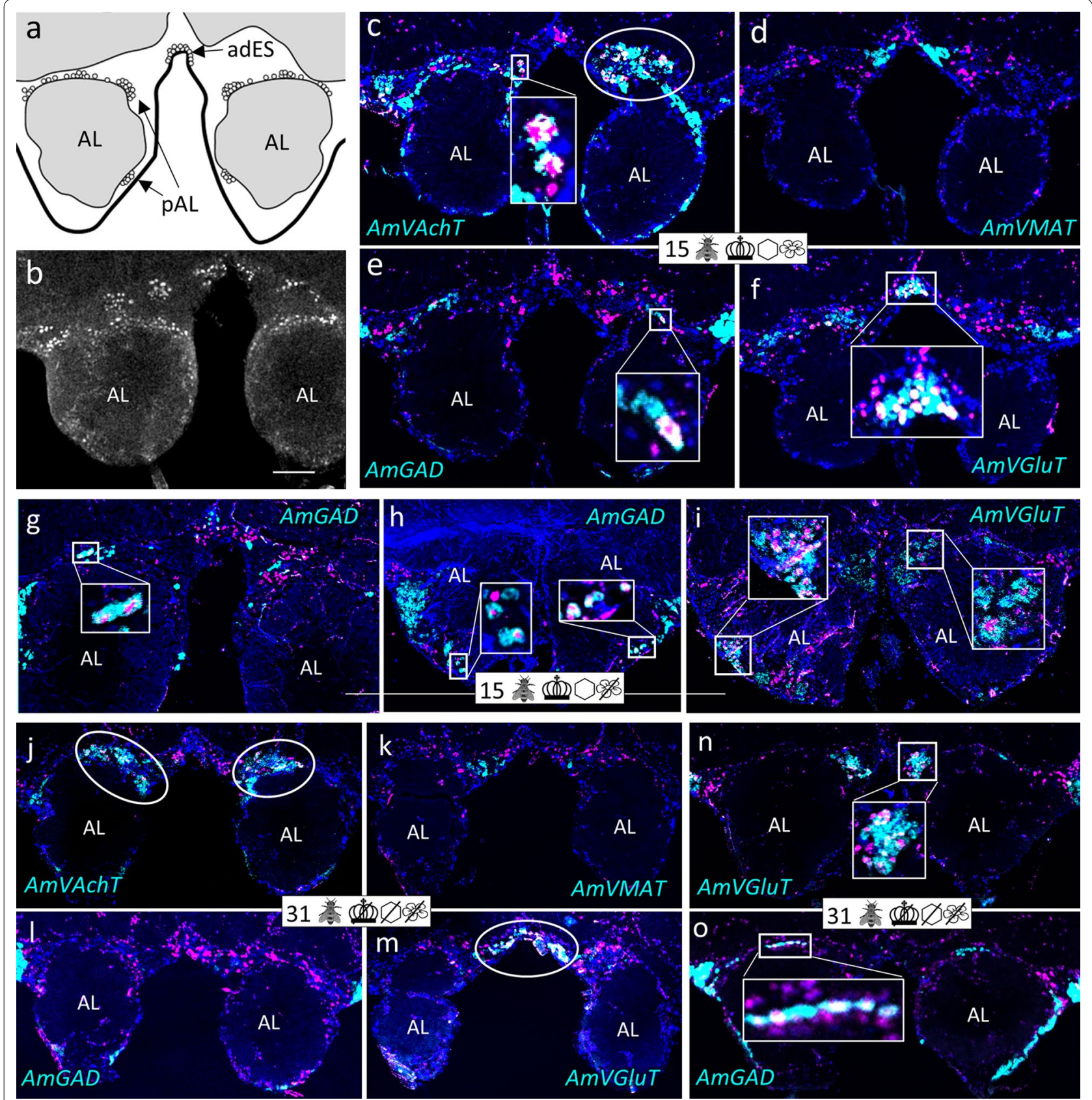

Fig. 3 Group 'around (peri) antennal lobe' (pAL) and cluster'anteriodorsal to the esophageal hole' (adES). a Schematic drawing of the honeybee AL. AmFoxP neurons are depicted as empty circles, arrows point to the two neuron populations. b Confocal image ( $2 \mu \mathrm{m}$ confocal section) of a pupal brain stained with the AmFoxP ${ }^{42 \mathrm{kDa}}$ antiserum (white label). $\mathbf{c}-\mathbf{o}$ dISH stainings show AmFoxP transcript (magenta), neurotransmitter marker transcript (cyan) and DAPI-stained nuclei (blue). Large rectangular insets show higher magnifications of small boxed regions, with white label reflecting co-expressing neurons. Some double-labeled neurons are also visible in circled areas in $\mathbf{c}, \mathbf{j}, \mathbf{m}$. c-f Adjacent cryosections of a 15 days old individual from the 'unmanipulated' condition. g-i Adjacent cryosections of a 15 days old individual from the 'honeycomb' condition, anterior (g) and posterior (h, i) . j-o Adjacent cryosections of a 31 days old individual from the 'incubator' condition, anterior (j-m) and posterior (n, $\mathbf{o})$. Pictograms refer to the conditions outlined in Table 1. Anatomical abbreviations see Table 5. Scale bar $\mathbf{b}$ : $100 \mu$ m, as orientation for all other panels 


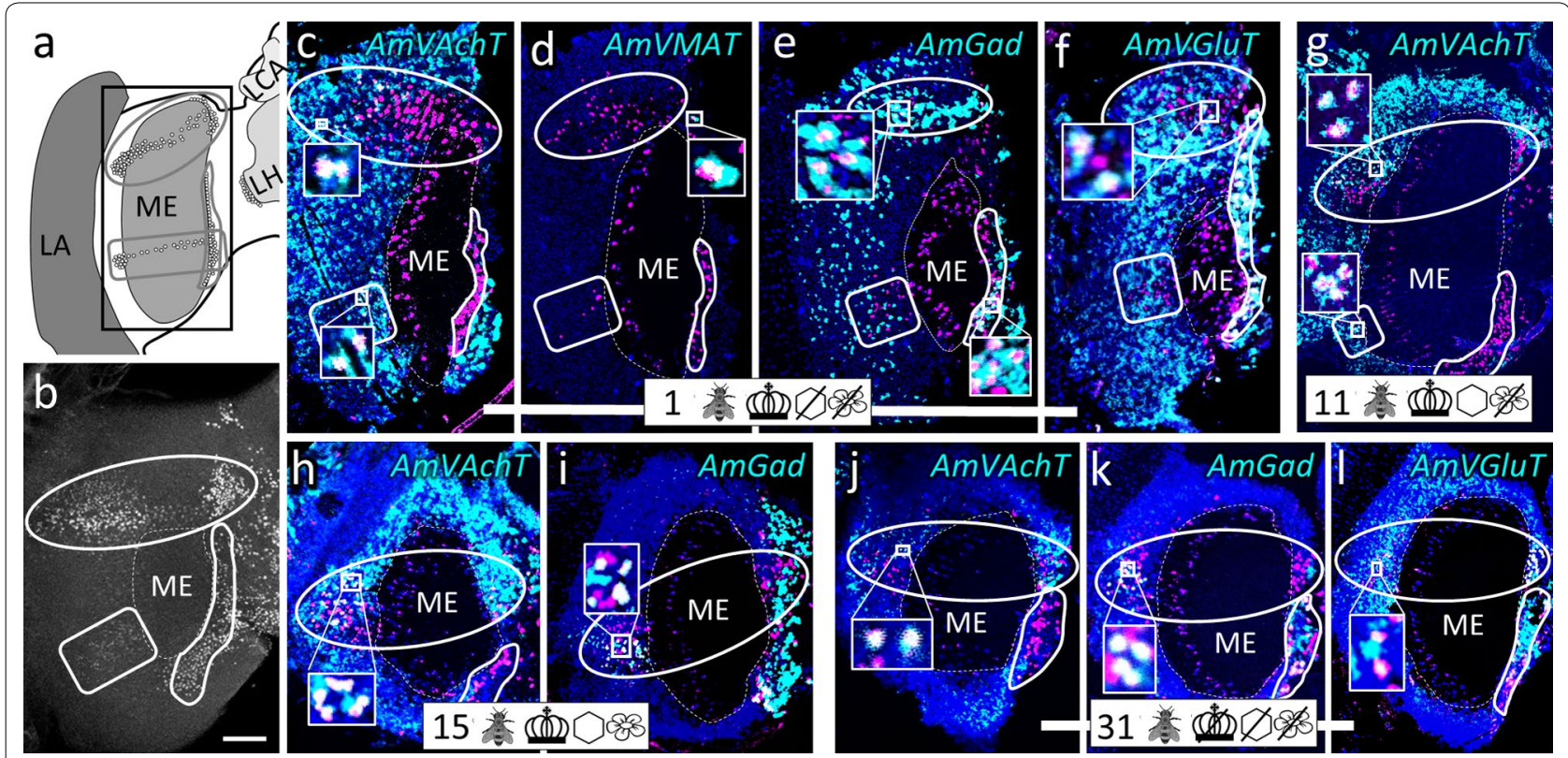

Fig. 4 Group'around (peri) medulla' (pME). a Honeybee brain schematic drawing focuses on the ME. AmFoxP neurons are depicted as empty circles which surround the ME in three subsets: a dorsal (gray circle) and a ventral (gray rectangle with rounded corners) subset wrap around the anterior ME, a third subset (outlined in gray) is bordering the ME medially in a thin strip. The black rectangle indicates the localization of panels (c-I). b Confocal image ( $60 \mu \mathrm{m}$ confocal stack) of a pupal brain stained with the AmFoxP ${ }^{22 k D a}$ antiserum (white label). The outlined areas refer to the AmFoxP subsets as in $\mathbf{a}$, the ME is indicated with a dashed line. c-I dISH stainings showing AmFoxP transcript (magenta), neurotransmitter transcript (cyan) and DAPI-stained nuclei (blue). Rectangular insets show co-localization of the two probes, resulting in white label. c-f Adjacent cryosections of a newly emerged individual. $\mathbf{g}$ Cryosections of an 11 days old individual from the 'honeycomb' group. $\mathbf{h}$, $\mathbf{i}$ Adjacent cryosections of a 15 days old individual from the 'unmanipulated'group. $\mathbf{j}$-I Adjacent cryosections of a 31 days old individual of the 'incubator' group. For explanations of pictograms see Table 1, for anatomical abbreviations see Table 5. Scale bar b: $100 \mu \mathrm{m}$, as orientation for all other panels

complex (dCX, Fig. 8h). AmVGluT-expressing AmFoxP neurons in the $\mathrm{dCX}$ were located medial to AmGadexpressing ones. The AmVGlut neurons in the pAL, pME and vGNG were intermingled with AmFoxP neurons expressing AmGad and AmVAchT.

\section{Monoaminergic AmFoxP neurons}

The monoaminergic marker VMAT labels neurons that express histamine (HA), octopamine (OA), dopamine (DA) or serotonin (5-HT). Large clusters were located in the region of the antennal commissure (Fig. 3e, i) and ventral to the lateral calyx (LCA) (Figs. 5d, 11b), likely corresponding to the dopaminergic $\mathrm{C} 1$ and $\mathrm{C} 3$ clusters previously described $[51,102,103]$. Further large $A m V$ $M A T$ positive cells were localized in the somatic region ventral to the GNG, probably corresponding to the octopaminergic VUMmx neurons described previously [53, 83, 103]. Only a few isolated AmFoxP neurons co-localized with AmVMAT. They did not belong to any of the above described neuron populations (Fig. 1; Table 3). Because there were only individual neurons co-labeled by AmFoxP and AmVMAT we decided to name them 'MF neurons'; monoaminergic AmFoxP neurons. They were detected in four regions: dorsolateral to the AL ('MF1'), anteriomedial to the medulla ('MF2'), dorsomedial to the lobula ('MF3) and lateral to the upper division of the central body (CBU), part of the CX ('MF4') (Figs. 4d, 11).

\section{AmFoxP neuron populations and co-expressed neurotransmitters were consistently detected in all sampled brains}

We qualitatively compared brains from individuals aged 1-50 days after emergence from the differently treated groups (Table 1). All 11 AmFoxP neuron populations were consistently detected, independent of age or treatment. The type of neurotransmitter being co-expressed was also stable and did not change (Figs. 3, 4, 5, 6, 7, 8, $9,10)$.

\section{Monoaminergic receptors in the $\mathrm{KC}$}

To further refine the identity of the AmFoxP-expressing $\mathrm{mKC}$ [32] we checked for co-expression of the D2-like dopamine receptor AmDop3 that was shown to be restricted to the IKC [150]. While the AmDop3 senseprobe (control) did not show any unspecific staining (Fig. 12f), our $d I S H$ stainings with the AmDop3 antisense probe revealed no coincidence with AmFoxP label within 


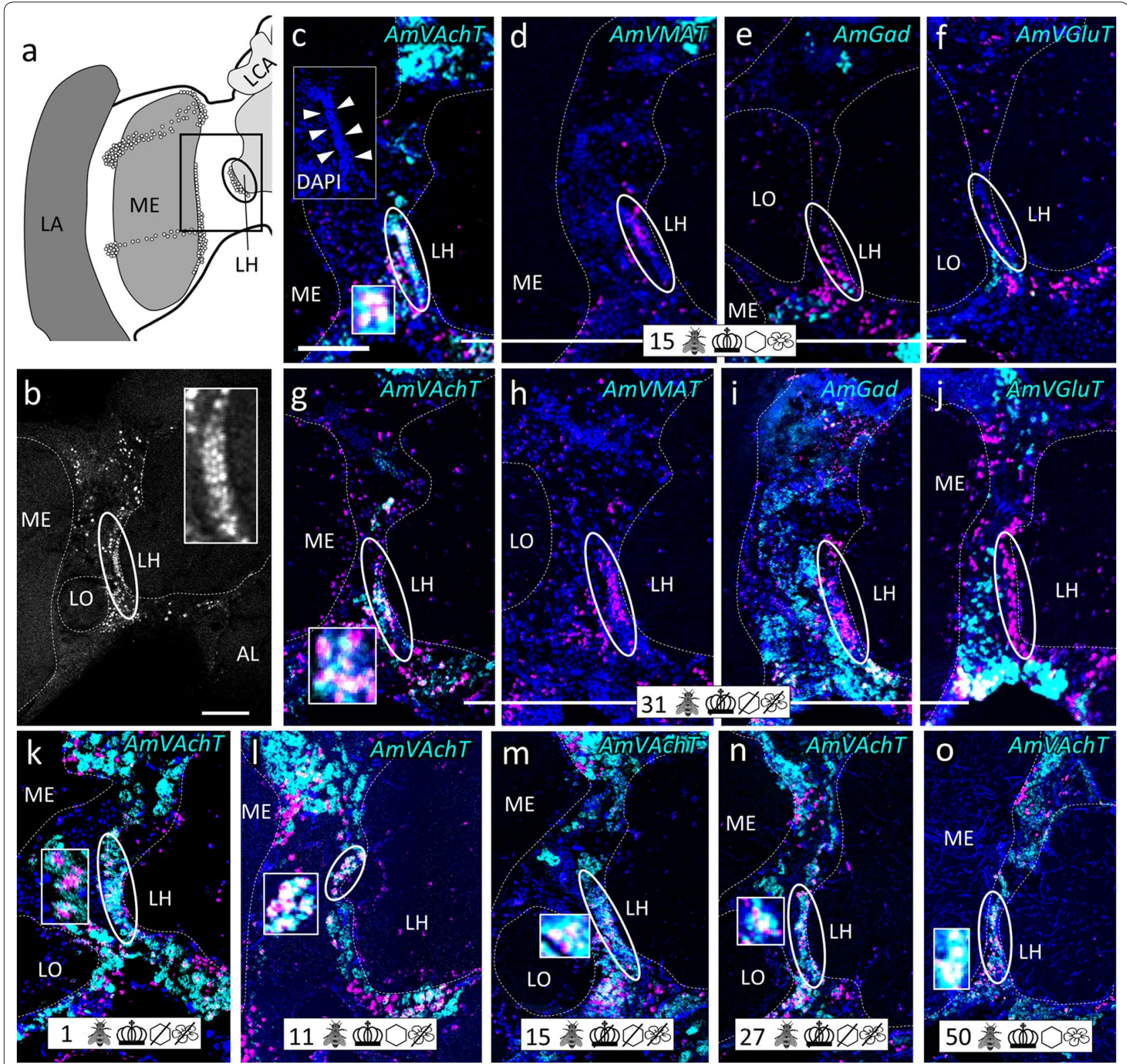

Fig. 5 Cluster'lateral to the lateral horn' (ILH). a Honeybee brain schematic drawing focuses on the LH and ME. AmFoxP neurons are indicated with small empty circles. Rectangle indicates the localization of panels (c-o). b Confocal image ( $2 \mu \mathrm{m}$ confocal section) of a forager brain stained with the AmFoxP ${ }^{42 \mathrm{KDa} a}$ antiserum (white label). c-o dISH stainings showing AmFoxP transcript (magenta), neurotransmitter transcript (cyan) and DAPI-stained nuclei (blue) on $12 \mu \mathrm{m}$ brain cryosections. Small rectangular insets in $\mathbf{c}, \mathbf{g}, \mathbf{k}$-o show co-localization of the two probes. Larger rectangular insets in $\mathbf{b}$, c show densely-packed small nuclei of the ILH, stained with AmFoxP42kDa antiserum (b) and DAPI (c). c-f Adjacent cryosections of a 15 days old individual from the 'unmanipulated' group. $\mathbf{g - j}$ - Adjacent cryosections of a 31 days old individual from the 'incubator' group. $\mathbf{k}$ Newly emerged individual. I 11 days old individual from the'honeycomb' group. $\mathbf{m} 15$ days old individual from the 'incubator' group. n 27 days old individual of the 'incubator' group. $\mathbf{o}$ Cryosection of a 50 days old individual from the 'unmanipulated' group. For explanations of pictograms see Table 1, for anatomical abbreviations see Table 5. Scale bar b, c: $100 \mu$ m, as orientation for all other panels

the same neurons, but juxtaposed populations of neurons expressing AmFoxP or AmDop3 (Fig. 12g).

Figure 13 schematically summarizes the neurotransmitter profiles of AmFoxP neuron populations in the honeybee brain described above.
AmVAchT and AmVGluT expression pattern in the KC varied in bee samples of different age

Our stainings consistently revealed AmVAchTexpression in the class I KC across all samples. This was confirmed by RT-qPCR data that revealed ten 


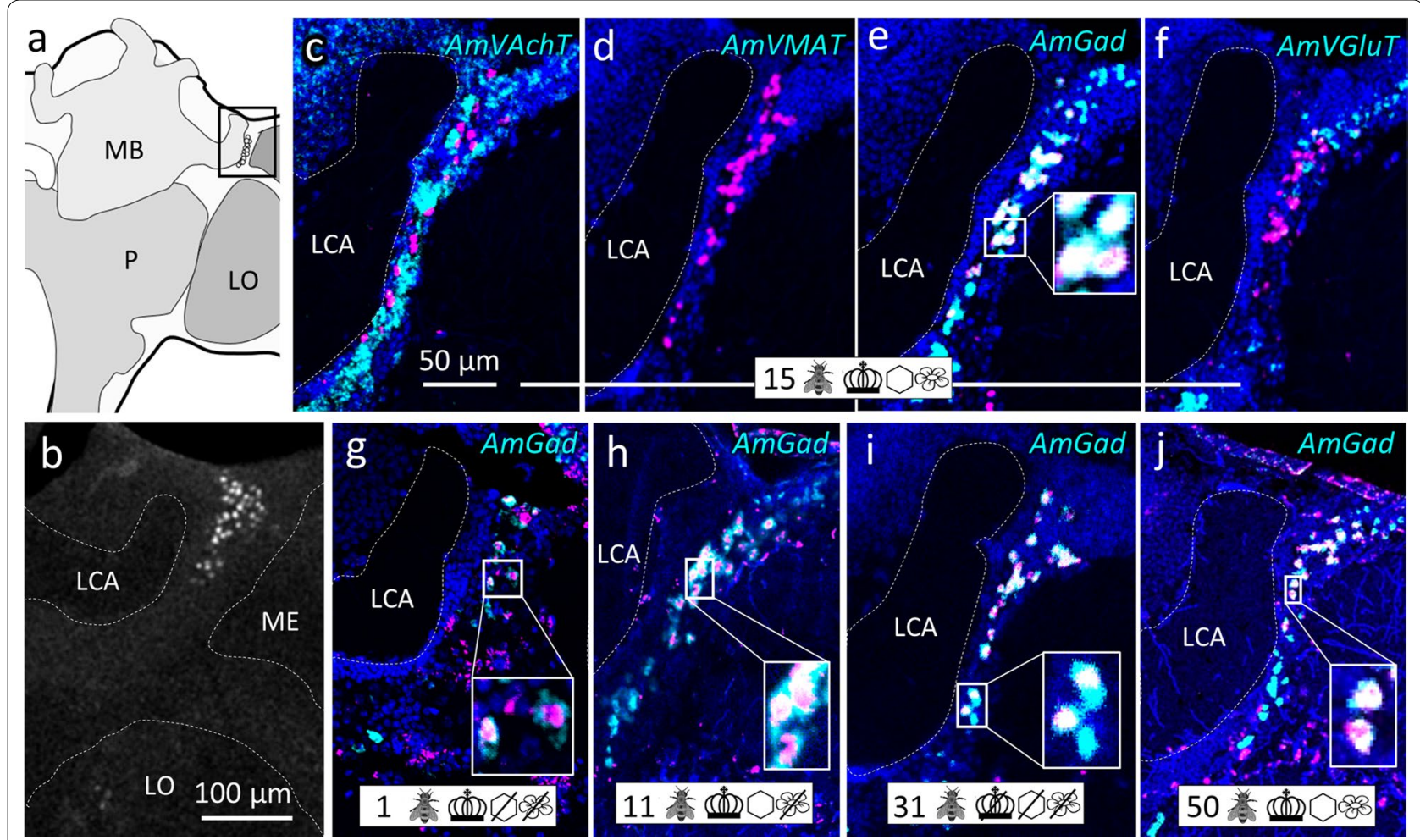

Fig. 6 Cluster anteriolateral to the lateral calyx (alLCA). a Schematic drawing of the medial level of one hemisphere. AmFoxP neurons are indicated by small empty circles within the boxed area which indicates the localization of panels $(\mathbf{b}-\mathbf{j})$. b Confocal image ( $2 \mu \mathrm{m}$ confocal section) of a pupal brain stained with the AmFoxP42kDa antiserum (white label). $\mathbf{c}-\mathbf{j}$ dISH stainings showing AmFoxP transcript (magenta), neurotransmitter transcript (cyan) and DAPI-stained nuclei (blue) on $12 \mu \mathrm{m}$ cryosections. Rectangular insets show magnified boxed area with co-localization of the two probes. c-f Adjacent cryosections of a 15 days old individual from the 'unmanipulated' group. $\mathbf{g}$ Cryosections of a newly emerged individual. $\mathbf{h}$ Cryosection of an 11 days old individual from the 'honeycomb' group. i Cryosections of a 31 days old individual of the 'incubator' group. $\mathbf{j}$ Cryosection of a 50 days old individual of the 'control'group. For explanations of pictograms see Table 1, for anatomical abbreviations see Table 5. Scale bars as indicated. Scale bar in c as orientation for the panels $(\mathbf{d}-\mathbf{j})$

times more AmVAchT than AmVGluT mRNA levels in extracts prepared from calyces (Fig. $14 \mathrm{~m}$ ). AmVMAT and AmVIAAT levels were very low compared to AmVAchT (AmVMAT: 100 times less, AmVIAAT: 70 times less).

Interestingly, in younger bees (age 1-4) AmVAchT expression was restricted to the small and middle $\mathrm{KC}$ (sKC and $\mathrm{mKC}$ ) (Figs. 2, 14a, b) whereas the large KC (IKC) only started expressing AmVAchT between the ages 11-15 (Fig. 14c, d). In very old bees (aged 40 and 50 days) the intensity of AmVAchT-expression in the sKC decreased but did no longer differ between the different types of class I KCs (Fig. 14e). Although very low in general and only visible with increased brightness and contrast (FIJI), AmVGluT expression levels in the small and medial $\mathrm{KC}$ were comparatively higher between 1 and 19 days after emergence than in older bees (Fig. 14f-j). Low AmVGluT levels in the KC were also detected with RT-qPCR and 7-11 times higher than AmVIAAT and AmVMAT levels (Fig. 14m).

\section{Discussion}

The transcription factors FOXP1, FOXP2 and FOXP4 are relevant for human cognition including language [104]. They are strongly conserved in vertebrates where they play a role in sensory processing, motor behaviors and learning [105-110]. The genomes of other animals harbor only a single FoxP gene locus encoding a gene with a highly conserved DNA-binding domain $[25,33,111$, 112]. The fact that the neural expression pattern in vertebrates and the Forkhead box sequence in bilateria are so highly conserved raises the question whether FoxP might bestow neurons with particular properties and functions that might have been conserved since the last common ancestor of the bilateria. This type of 'deep homology' has been noted for a number of other transcription factors [90, 91, 113-116]. Alternatively, honeybee FoxP (AmFoxP) might have very different functions than vertebrate FoxPs, depending on the evolution of the FoxP gene's regulatory sequences and differences in binding of 


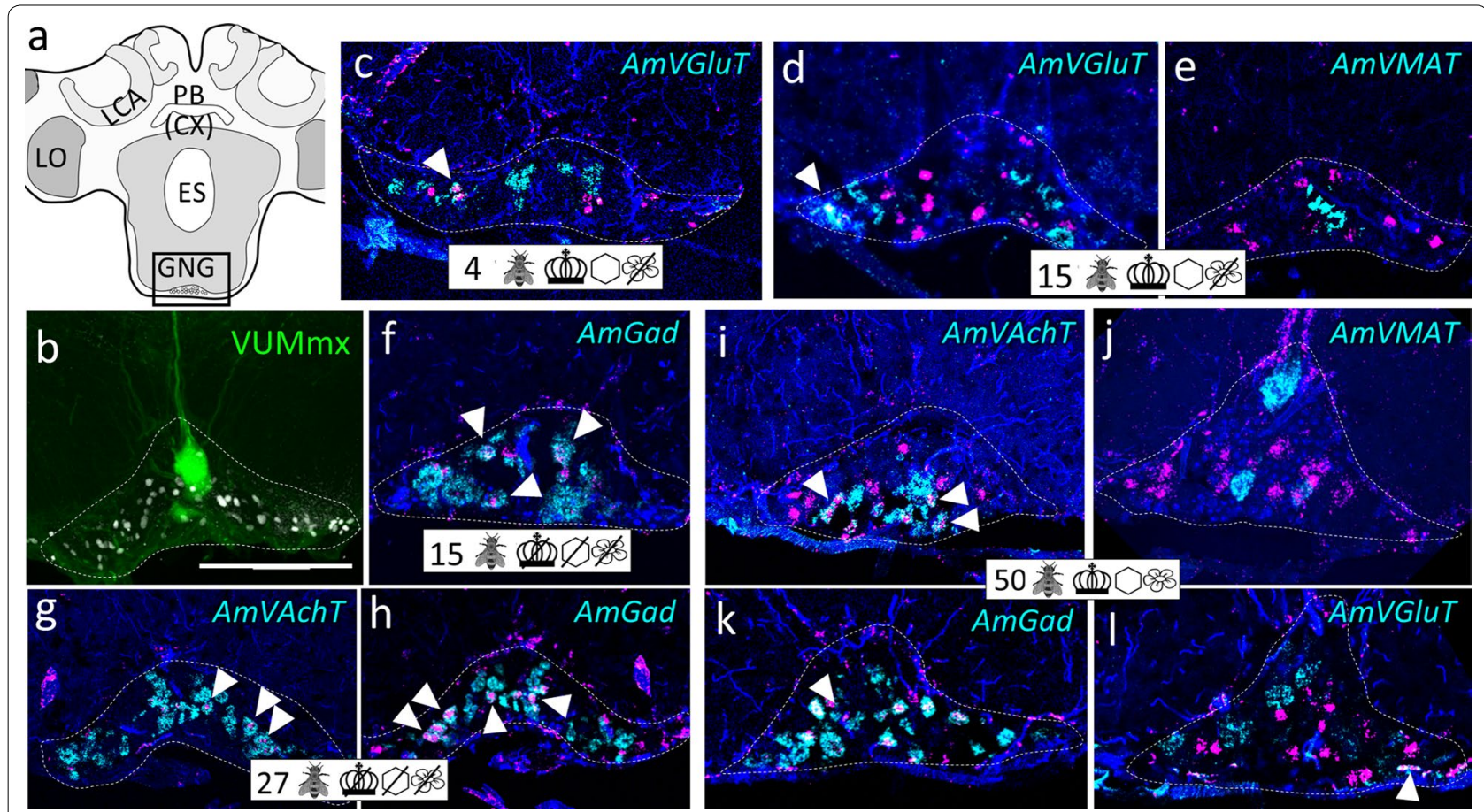

Fig. 7 Group ventral to the GNG (vGNG). a Honeybee brain scheme focusing on the posterior brain. AmFoxP neurons are indicated with white circles within the boxed area which indicates the localization of panels (b-I). b Confocal image (60 $\mu \mathrm{m}$ confocal stack) of a forager brain backfilled with lucifer yellow (green) and subsequently stained with the AmFoxP ${ }^{42 \mathrm{KDa}}$ antiserum (white label). The large green-labeled neuron lying on the brain midline is a VUMmx neuron. c-I dISH stainings show AmFoxP transcript (magenta), neurotransmitter transcript (cyan) and DAPI-stained nuclei (blue) on $12 \mu \mathrm{m}$ cryosections. White arrowheads point to neurons with co-localized probe signal. c Cryosections of a 4 days old individual from the 'honeycomb' group. d, e Adjacent cryosections of a 15 days old individual from the 'honeycomb' group. f Cryosections of a 15 days old individual from the 'incubator' group. $\mathbf{g}$, $\mathbf{h}$ Adjacent cryosections of a 27 days old individual from the 'incubator' group. i-I Adjacent cryosections of a 50 days old individual from the 'control'group. For explanations of pictograms see Table 1, for anatomical abbreviations see Table 5. Scale bar b: $100 \mu$ m, as orientation for all other panels

the FoxP proteins to their target genes and other proteins [117].

As a first step towards the evaluation of these hypotheses, we used bee brains to map the distribution of isoform-specific AmFoxP expressing neurons and for some of them their projection pattern [25]. AmFoxP is expressed in particular sets of neurons in most cortical regions of the honeybee brain, many of them densely clustered, whereas others are found in a more distributed fashion [25].

In the present report we classified AmFoxP neurons by their neurotransmitters without discriminating between the two previously described AmFoxP isoforms. We examined individuals of different age and treatments to qualitatively assess whether the number of populations was rather robust or susceptible to different sensory and motor experience. FoxP2 can be regulated developmentally as well as by motor activity and sensory stimuli [13, 14, 47-49]. Overall, we found that AmFoxP neurons in the honeybee co-expressed a variety of neurotransmitters, similar to what has been reported in vertebrates.
Below we will discuss the transmitter profile of different neurons in honeybee and other insect brains based on the markers we used, and propose how these neurons might relate to already described neurons and their function.

\section{Neurotransmitters}

Neurotransmitters are essential for the identity and function of neurons. In honeybees their expression levels have been correlated to certain behaviors, e.g. novelty-seeking [118] or learning and memory [119]. Some of the proteins involved in neurotransmitter release are conserved and date back to the last common ancestor of eukaryotes [120,121]. We focused on the most abundant neurotransmitters in the insect brain, i.e. glutamate, GABA, acetylcholine and monoamines. Using double-label in situ hybridization we revealed differential expression of their markers in AmFoxP neuron populations. The seven previously defined AmFoxP neuron clusters (adES, ILH, alLCA, vMCA, mKC, plLCA, mvLO) co-expressed only one of the four neurotransmitter markers whereas the four groups (pAL, 

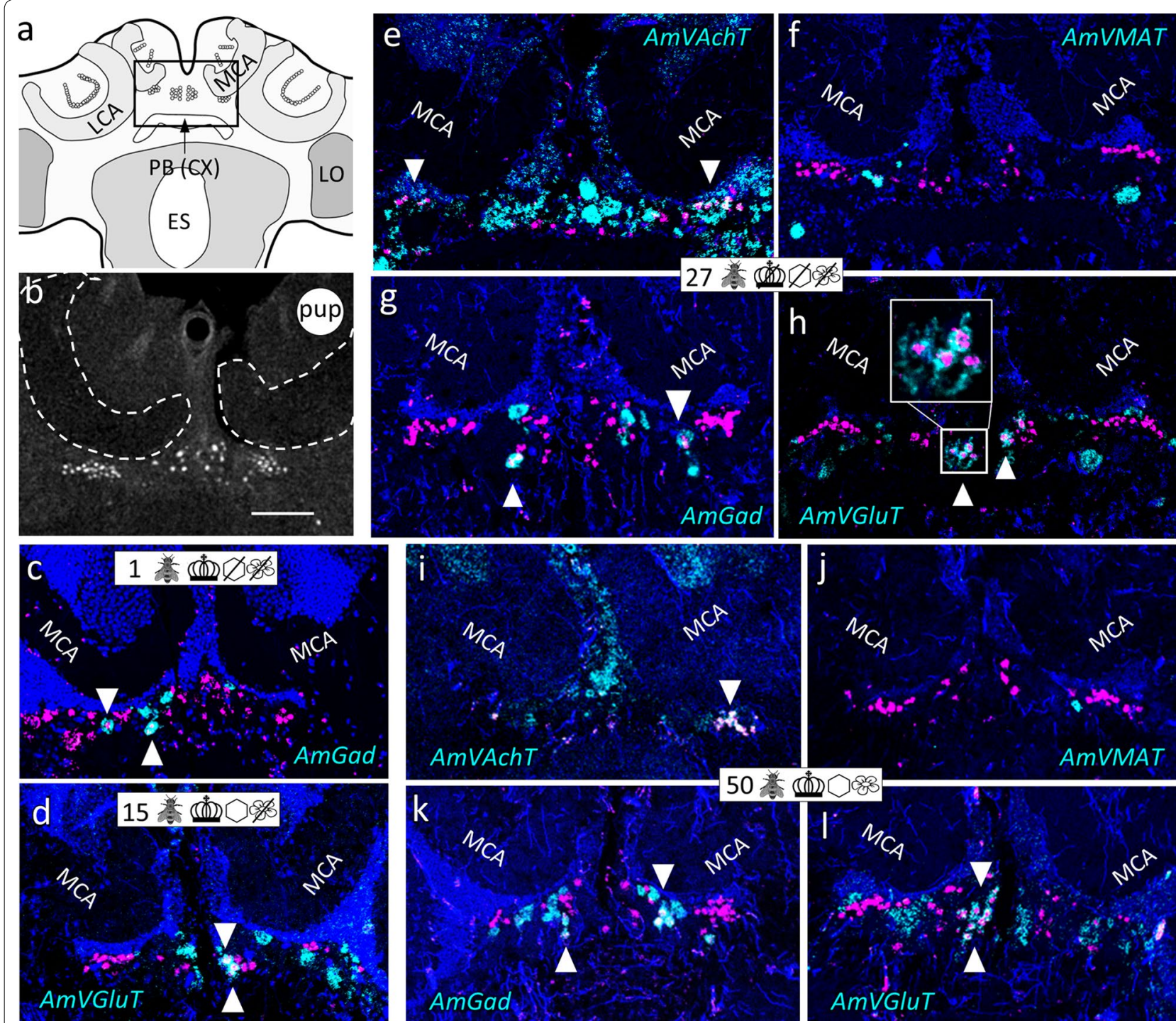

Fig. 8 Cluster ventral to the medial calyx (vMCA) and group dorsal to the central complex (dCX). a Honeybee brain schematic drawing focuses on the posterior brain. AmFoxP neurons are depicted as small empty circles. Rectangle indicates localization of panels (b-l). $\mathbf{b}$ Confocal image $(2 \mu \mathrm{m}$ confocal section) of a pupal brain stained with the AmFoxP $42 \mathrm{kDa}$ antiserum (white label). c-I dISH stainings show AmFoxP transcript (magenta), neurotransmitter transcript (cyan) and DAPI-stained nuclei (blue) on $12 \mu \mathrm{m}$ cryosections. Arrowheads point to neurons that co-express AmFoxP and a neurotransmitter. c Cryosection of a newly emerged individual. $\mathbf{d}$ Cryosection of a 15 days old individual from the 'honeycomb' group. e-h Adjacent cryosection of a 27 days old individual from the 'incubator' group. i-I Cryosection of a 50 days old individual from the 'control' group. For explanations of pictograms see Table 1, for anatomical abbreviations see Table 5. Scale bar $\mathbf{b}$ : $100 \mu \mathrm{m}$, as orientation for all other panels

pME, dCX, vGNG) showed a rather heterogeneous neurotransmitter profile. This finding corroborates the differentiation between the two AmFoxP neuron population classes, i.e. 'groups' and 'clusters'. Within the 'groups' there might be smaller subunits that project to the same areas and co-express only one neurotransmitter. The pAL could certainly be further subdivided into smaller clusters lateral, medial, ventral and dorsal to the $\mathrm{AL}$, being glutamatergic, GABAergic or cholinergic.

\section{Acetylcholine}

Three of the four AmFoxP groups (pAL, pME, vGNG) and five of the seven clusters (ILH, mvLO, plLCA, vMCA, mKC) expressed AmVAchT. The largest cluster, the mvLO, consists of two divisions, an anterior $(\mathrm{mvLO}-\mathrm{a})$ and a posterior (mvLO-p) part that project to different areas of the brain [25]. The neurites of the mvLO-p somata converge onto a tract that connects the lobula with the posteriolateral protocerebrum 


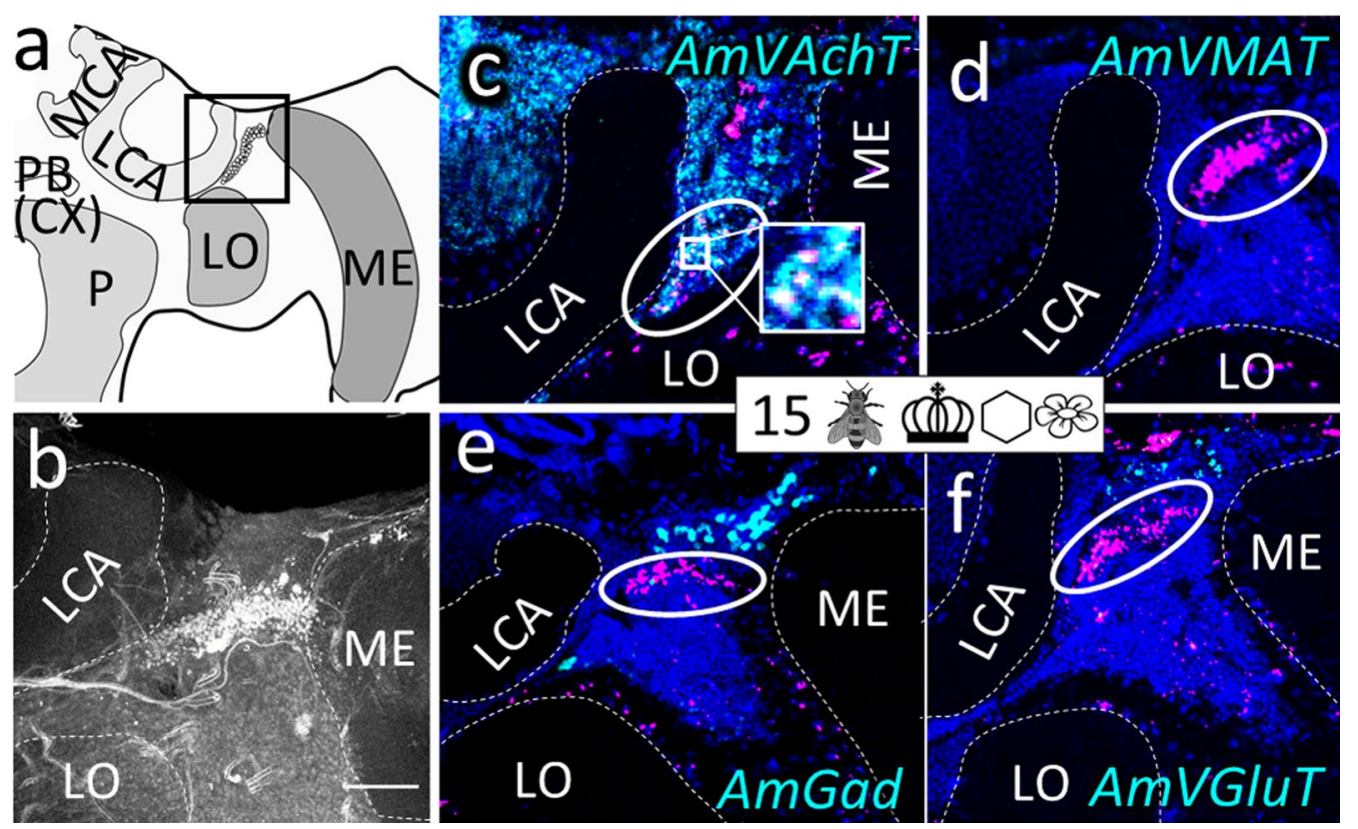

Fig. 9 Cluster posteriolateral to the lateral calyx (pILCA). a Honeybee brain schematic drawing of the right hemisphere in the posterior brain. AmFoxP neurons are indicated with small white circles within the boxed area which indicates the localization of panels ( $\mathbf{b}-\mathbf{f}$ ). $\mathbf{b}$ Confocal image ( $60 \mu \mathrm{m}$ confocal stack) of a forager brain stained with the AmFoxP ${ }^{22 \mathrm{KDa} a}$ antiserum (white label). $\mathbf{c}-\mathbf{f}$ dISH stainings show AmFoxP transcript (magenta), neurotransmitter transcript (cyan) and DAPI-stained nuclei (blue) on $12 \mu \mathrm{m}$ adjacent cryosections of a 15 days old individual from the 'unmanipulated' group. Inset in c shows magnification of small boxed area with co-localization of the two probes. For explanations of pictograms see Table 1, for anatomical abbreviations see Table 5. Scale bar b: $100 \mu \mathrm{m}$, as orientation for all other panels

(PLP). Such a posterior cholinergic tract was previously seen with AChE histochemistry [56]. Whether the GABAergic, AmFoxP-negative neurons between the two subdivisions of the cluster converge onto the same tracts as the mvLO-a or mvLO-p requires further studies. Schäfer and Bicker [50] showed a GABAir tract connecting the posterior part of the lobula and the protocerebrum that could be intermingled with the AchE-tract. If this were the case the PLP would receive inhibitory and excitatory input from the lobula. The circuitry involved might be thus important to extract oscillating, like temporal features of sensory signals [122-124].

The cholinergic vMCA is reminiscent of a cluster with AchE activity ventral to the medial calyx and dorsal to the anterior optic tract (AOT) described by Kreissl and Bicker [56]. This cluster connects to the fan-shaped body/ $\mathrm{CBU}$, a major subdivision of the central complex which is important for polarized vision, motor control and spatial memory $[125,126]$. In future studies, neuronal tracing will help to further identify the other two cholinergic AmFoxP clusters; i.e. the ILH and the plLCA.

Interestingly, AmVachT was highly expressed in the $\mathrm{KC}$, as shown by both RT-qPCR and $d I S H$. Staining was punctate and coincided less with cell bodies, in contrast to the AmVachT staining observed in the rest of the brain. Because we do not assume local translation and the puncta were numerous we do not think that these signals are located synapses contacting the $\mathrm{KC}$ somata which were described only for dopaminergic synapses [127]. Due to the concentrated and punctuate staining pattern it is difficult to determine whether AmFoxP and AmVachT co-localized in all mKC. Based on our analysis (see "Cholinergic AmFoxP neurons" section) we conclude that they predominantly do. Until recently there was no strong evidence about the identity of $\mathrm{KC}$ neurotransmitters, one of the main limitations being the difficulty to isolate postsynaptic neurons to $\mathrm{KC}$ to characterize physiologically their neurotransmitter profiles. Previous studies in the honeybee described the expression of Acetylcholinesterase (AchE) in KC [56, 128]. Shapira et al. [128] showed expression in all class I KC in nurses, but restricted signal in the $\mathrm{IKC}$ in foragers. AmVachT expression in our study was restricted to the sKC and $\mathrm{mKC}$ in young bees and expanded to all class I KC in older bees. These differences could be explained by the different biosynthetic origins of the AchE enzyme and the vesicular transporter VAchT. AchE is associated to the neurotransmitter cycle by degrading acetylcholine in the synaptic cleft. In vertebrate neuromuscular junctions it can be 

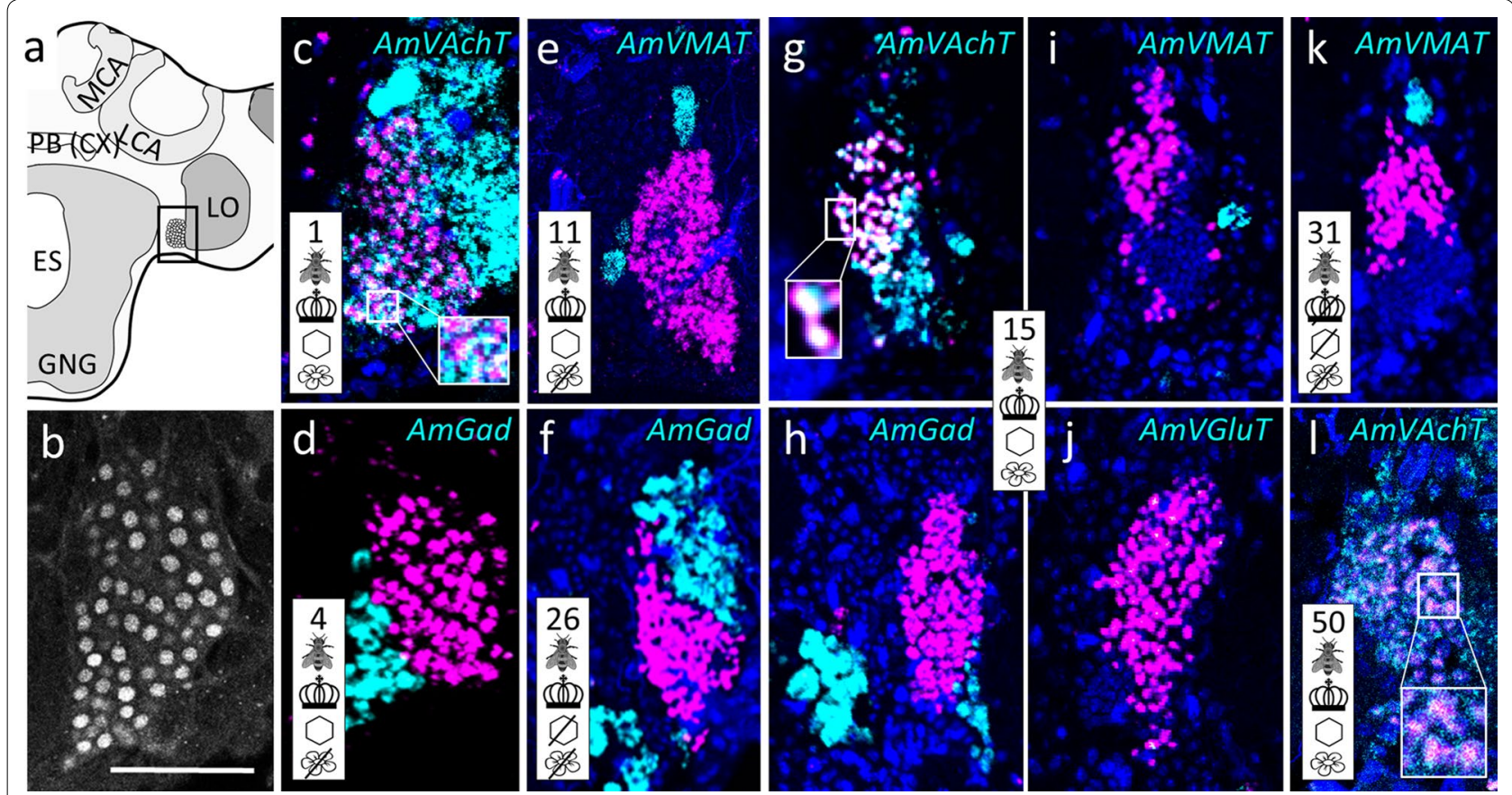

Fig. 10 Cluster medioventral to the lobula-posterior part (mvLO-p). a Honeybee brain schematic drawing focuses on a posterior brain hemisphere. AmFoxP neurons are indicated by small empty circles within the boxed area which indicates localization of panels $(\mathbf{b}-\mathbf{j})$. $\mathbf{b}$ Confocal image $(2 \mu \mathrm{m}$ confocal section) of a forager brain stained with the AmFoxP ${ }^{42 k D a}$ antiserum (white label) shows the mvLO-p. c-I dISH stainings showing AmFoxP transcript (magenta), neurotransmitter transcript (cyan) and DAPI-stained nuclei (blue) on $12 \mu \mathrm{m}$ cryosections. c Cryosection of a newly emerged individual. $\mathbf{d}$ Cryosection of a 4 days old individual from the 'honeycomb' group. e Cryosection of a 11 days old individual of the 'honeycomb' group. f Cryosection of a 26 days old individual of the 'mini-cage' group. 9-j Adjacent cryosections of a 15 days old individual from the 'unmanipulated' group. $\mathbf{k}$ Cryosection of a 31 days old individual of the 'incubator' group. I Cryosection of a 50 days old individual of the 'unmanipulated' group. Inset in $\mathbf{c}, \mathbf{g}$, I show magnification of boxed area with co-localization of the two probes. For explanations of pictograms see Table 1, for anatomical abbreviations see Table 5. Scale bar b: $50 \mu \mathrm{m}$, as orientation for all other panels
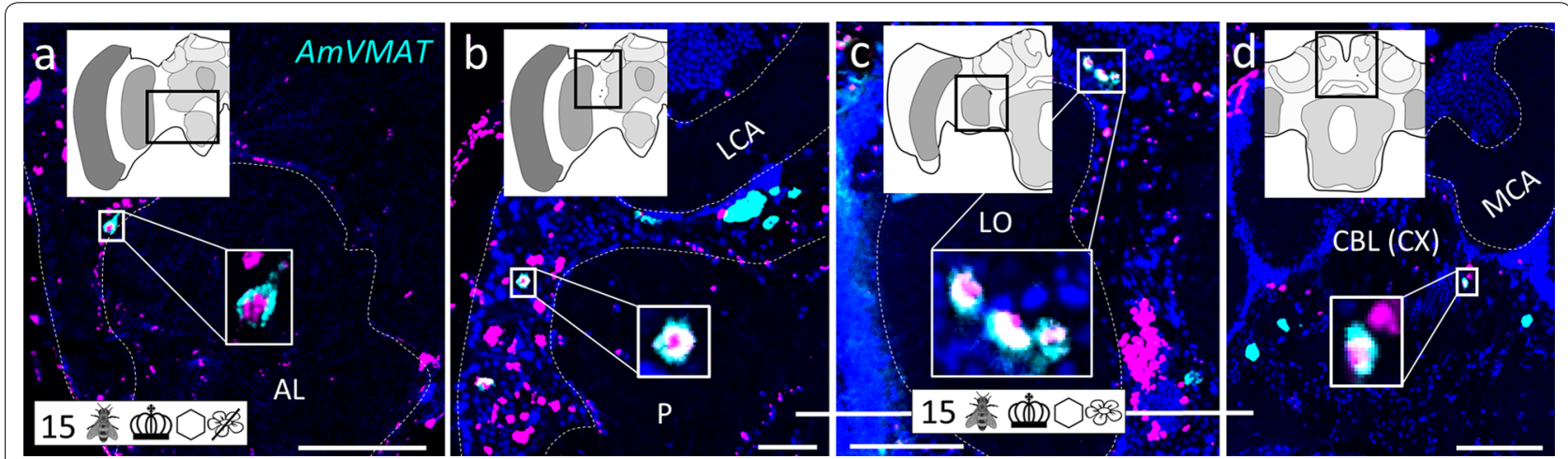

Fig. 11 Individudal 'MF' neurons (monoaminergic AmFoxP neurons) that co-express AmFoxP and AmVMAT. a-d dISH stainings showing AmFoxP transcript (magenta), AmVMAT (cyan) and nuclei (blue) in honeybee brain cryosections, anterior (a) to posterior (d). Insets outlined by white boxes show co-localization of the two probes. Locations in the brain where photomicrographs were taken are outlined (black line) in the schematic drawing in the insets at the top of each panel. a'MF1'neurons dorsolateral to the antennal lobe (AL) of a 15 days old individual from the'encaged honeycomb' group. b 'MF2' neuron medial to the medulla (ME). c'MF3' neurons dorso-medial to the lobula (LO). d'MF4' neurons close to the fan-shaped body (FB) of the central complex (CX). b-d Cryosections of a 15 days old individual from the 'control'group. For explanations of pictograms see Table 1, for anatomical abbreviations see Table 5. Scale bars: $100 \mu \mathrm{m}$ 


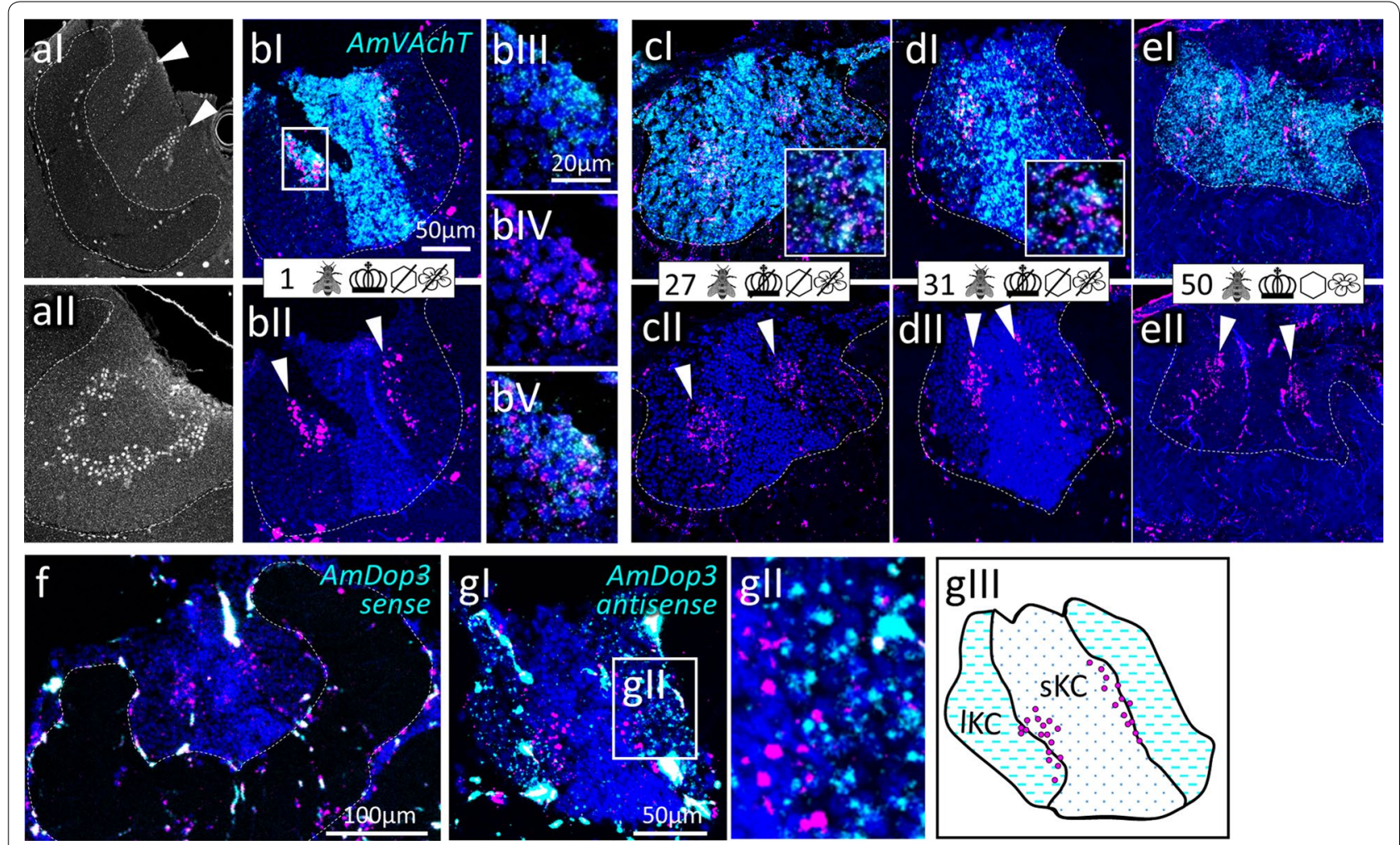

Fig. 12 AmFoxP expressing Kenyon cells (KC) of the mushroom bodies. a Single confocal sections ( 1 um) show the KC of a whole-mount pupal brain stained with the AmFoxP $42 \mathrm{kDa}$ antiserum (white label), (al) anterior, (all) posterior. The calyx is encircled with a dashed line. White arrowheads point to the elongated AmFoxP neuron clusters (mKC). b-e ISH stainings show AmFoxP transcript (magenta) and AmVAchT transcript (cyan) on $12 \mu \mathrm{m}$ cryosections of individuals taken from the different treatment groups. Age and treatment group are indicated with symbols that can be seen in Table 1. Magnifications of inset in $\mathbf{b}$ is shown in panels (bIll-V). The two transcripts of AmFoxP and AmVAchT are mostly co-expressed in the same cells. f, $\mathbf{g}$ dISH stainings show expression of the D2-like dopamine-receptor Amdop3 (cyan) and AmFoxP (magenta). The Amdop3 sense probe (control) did not show any (unspecific) staining (f). Amdop3 is mostly expressed in the IKC but there was a small overlap with the AmFoxP-expressing mKC (gl-glil). Inset in $\mathbf{g l}$ is shown magnified in gll. glll shows a schematic of gl. Blue: nuclear staining (DAPI). Sizes of scale bars are indicated

released pre- and post-synaptically [129] which makes it a marker for cholinergic as well as cholireceptive neurons [130]. Also, it is implicated in other metabolic pathways [131, 132]. Thus AchE produced by the KC might be predominantly transported to the microglomeruli in the calyx neuropil to hydrolyze Ach transmitted by projection neurons. Barnstedt et al. [133] demonstrated that some KC are cholinergic in Drosophila and our finding constitute the first unequivocal evidence that $\mathrm{KC}$ are cholinergic in the honeybee.

\section{Glutamate}

All four AmFoxP groups and one cluster, the adES, expressed AmVGluT. In VGlut-reporter Drosophila lines, several large glutamatergic neurons with a similar distribution as the neurons of the honeybee $\mathrm{dCX}$ cluster were shown to project into the central complex (CX) [134]. Thus, the AmFoxP neurons of the dCX might be a subset of glutamatergic neurons also projecting into the CX. This proposition is supported by the study on Drosophila
dFoxP gene activity in the CX by Lawton et al. [27]. As the CX neuropils strongly express GluCl receptors [64], the glutamatergic AmFoxP neurons of the dCX might transmit inhibitory input. Based on location, the glutamatergic AmFoxP neurons in the vGNG cluster might be descending (motor) neurons, possibly innervating neck muscles [135, 136].

In some sections, especially in newly emerged bees, sparse and very weak signals of AmVGluT were detected in the sKC. This was corroborated by the RT-qPCR data showing that the $\mathrm{KC}$ expressed small amounts of $A m V$ GluT, ten times less than AmVAchT, but 7-11 times more than AmVIAAT and AmVMAT. The very low levels of the latter two markers might be due to contamination of the samples with cells other than KC. Very weak glu-ir signals [52] and high mRNA amounts of AmEAAT [59] in the sKC of the MB have been described before $[52,59]$. In crickets, glu-like-ir signal was reported only in the (inner) KII and (outer) K III but not in the (inner) KI KC [54]. In Drosophila, glu-like-ir was detected in 


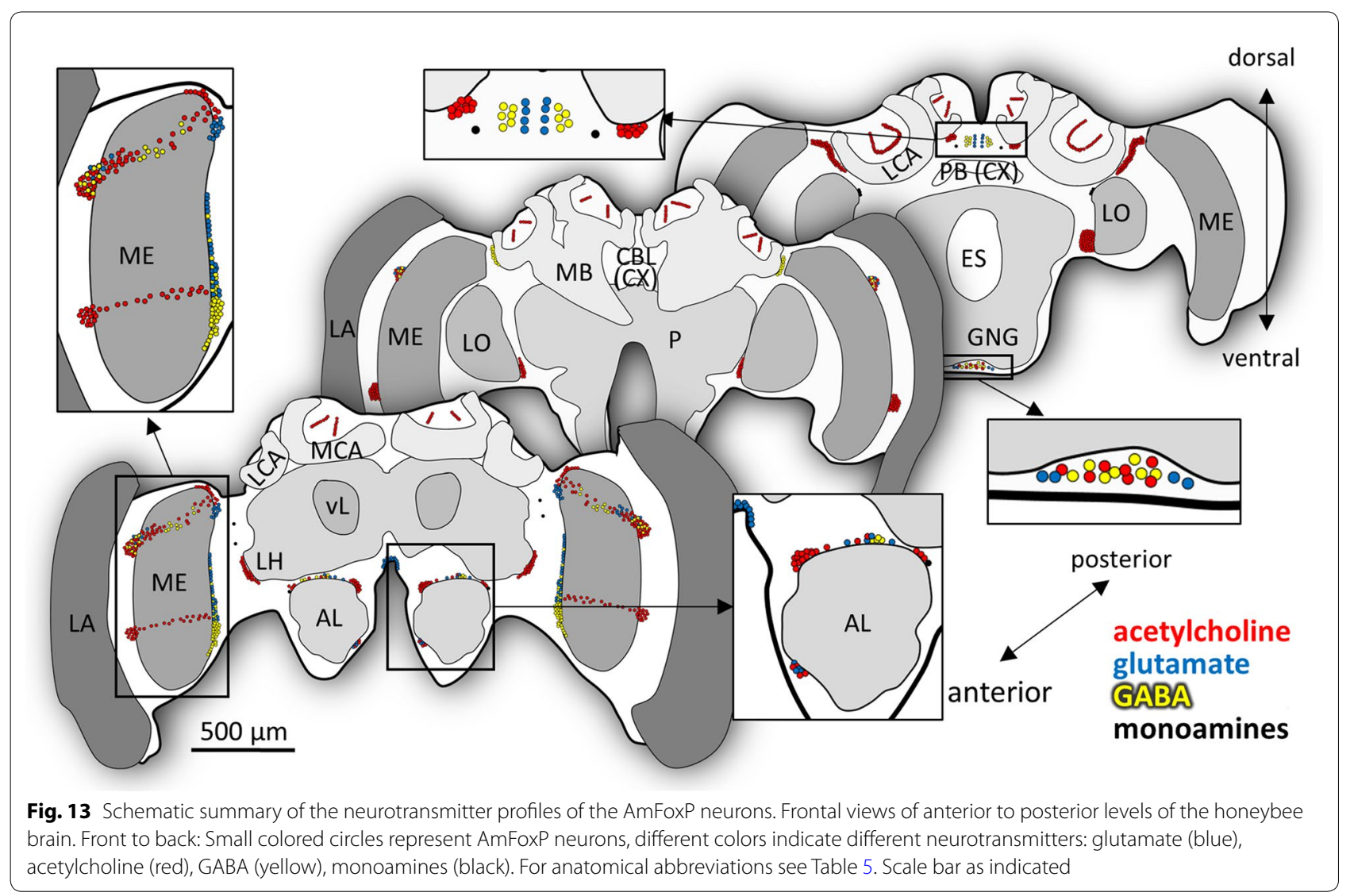

the $\alpha \beta_{c}-K C$ subset, which is developmentally generated last and expresses dFoxP [28, 32], but not dVGluT [55]. These authors discuss that neurotransmitter expression in the $\mathrm{KC}$ could vary over lifetime and that glutamate might be expressed only transiently. This is in line with our data, as we see weak signals in younger bees at the age of 1-19 days, but less distinctive or not at all in older bees, aged 23-50 days. This age-dependent difference could mirror the behavioral switch from nurses to foragers which is accompanied by gene expression differences [137].

\section{GABA}

The four AmFoxP groups and the alLCA cluster express AmGad. AmFoxP neurons dorsal to the CX (dCX) that co-express AmGad might correspond to neurons described by GABA-like-ir [50] and project to the CX. If the glutamatergic AmFoxP neurons connected to postsynaptic neurons with $\mathrm{GluCl}$ receptors in the CX, as mentioned above, then the $\mathrm{dCX}$ cluster would transmit only inhibitory input to the CX. Strausfeld and Hirth [91] have compared the CX to the vertebrate basal ganglia, based on similar developmental gene expression profiles, similar function in the selection and maintenance of adaptive behavior and because both constitute a 'midline brain structure' [91]. Interestingly the striatum of the basal ganglia express FoxP1, 2 and 4 [14, 21, 23, 138-140]; and plays a role in adaptive sensorimotor behavior [141-144]. Similarly, the CX is important for sensorimotor integration and motor control [91, 145-147] and it will be interesting for future studies to examine a possible corresponding role of the inhibitory AmFoxP neurons that might project to the CX.

The single AmGad-expressing AmFoxP neurons in the pAL neuron group are located anterio-dorsal and posterio-ventral to the AL but not in the larger AmGad-labeled clusters lateral to the AL which are most likely inhibitory projection neurons [94, 101]. The GABAergic AmFoxP neurons within the pAL might be local interneurons $[57,148]$. We also showed that the AmFoxP neurons in the mvLO are not GABAergic and thus confirmed that they do not correspond to the (more anteriorly located) A3v cluster as suggested by Kiya et al. [31]. 

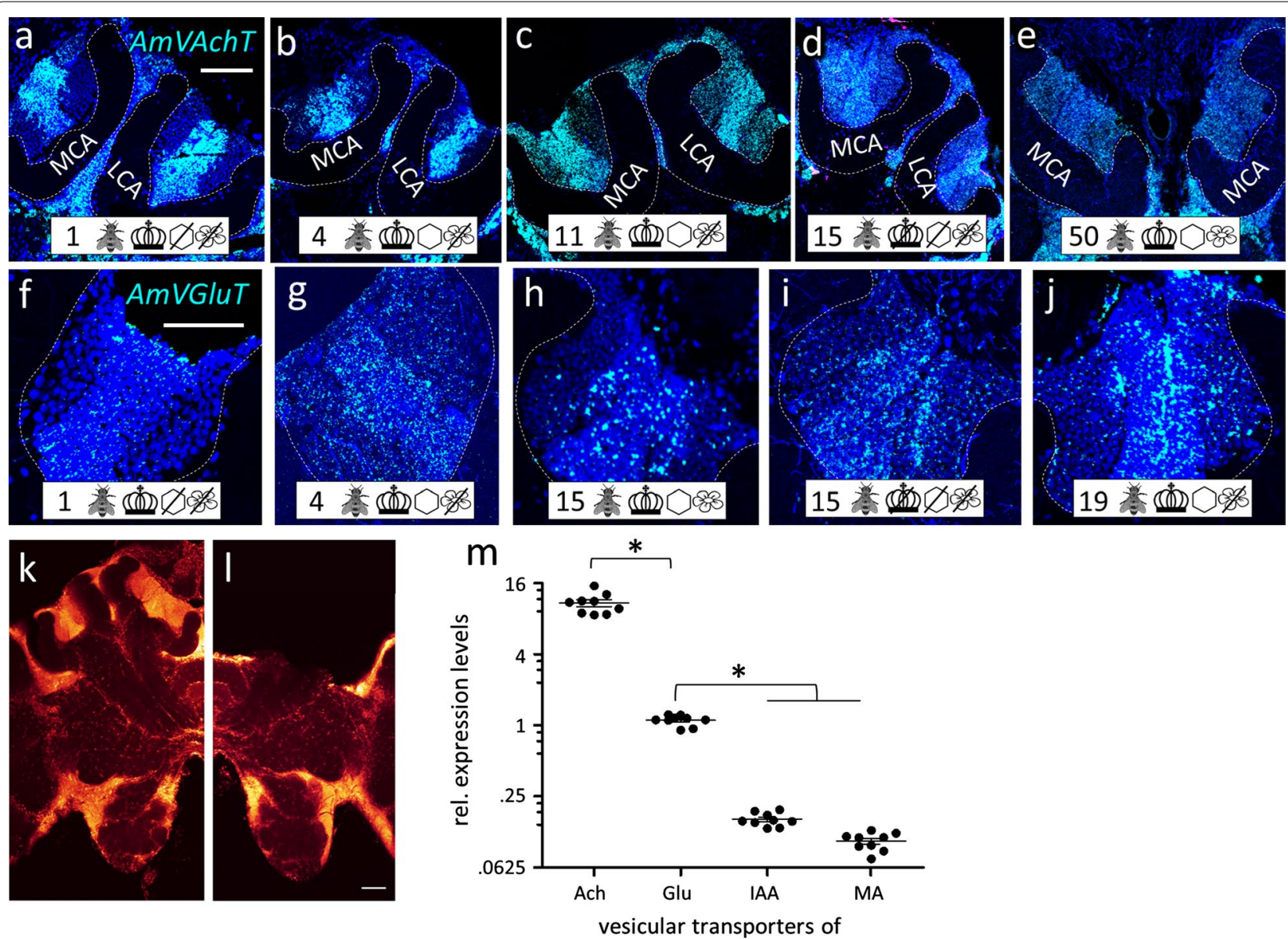

Fig. 14 AmFoxP and AmVGIUT mRNA expression in the KC. $\mathbf{a}-\mathbf{j}$ ISH stainings on $12 \mu \mathrm{m}$ cryosections of individuals taken from the different treatment groups (symbols, Table 1) show AmVAchT (a-e) and AmVGluT (f-j) expression in different KC subpopulations. AmVAchT was restricted primarily, in workers aged younger than 5 days, to the sKC and $\operatorname{mKC}(\mathbf{a}, \mathbf{b})$. With increasing age, the IKC also started expressing AmVAchT (c-e). Simultaneously, the expression intensity in the sKC decreased until, in a very old forager (50 days) there was no more difference between the sKC/mKC and the IKC detectable (e). $\mathbf{f}-\mathbf{j}$ Very low AmVG/uT signals in the sKC/mKC were detected in almost half of the samples (all shown here). Age and treatment groups are indicated with symbols that can be seen Table 1. k. I Two different forager brains (100 $\mu \mathrm{m}$ sections) before (k) and after (I) calyx dissection for RT-qPCR experiments (nuclear staining with SYTOX green). Scale bars: $100 \mu \mathrm{m}$, in $\mathbf{a}, \mathbf{f}$ as orientation for the panels (b-e) and (g-j). $\mathbf{m}$ Relative expression levels of vesicular transporters in calyx extracts, measured by RT-qPCR. In adult foragers AmVAchT levels were ten times higher than AmVGluT levels (paired Student's $\mathrm{t}(8)=13.1, p<.0001$ ). AmIAAT and AmVMAT levels were 7-11 times lower than AmVGIUT levels (AmVIAAT: paired Student's $\mathrm{t}(8)=30, p<.0001$, AmVMAT: paired Student's $\mathrm{t}(8)=27.4, p<.0001$ )

\section{Monoamines}

We found overlap between AmVMAT and AmFoxP expression only in less than ten isolated $A m V M A T$ expressing AmFoxP neurons throughout the brain whereas none of the eleven described AmFoxP neuron populations expressed $A m V M A T$. The isolated $A m V$ $M A T$-expressing neurons around the mvLO might be part of the $\mathrm{S}_{\mathrm{L}}$ cluster that projects dorsally [51, 102]. The MF1 might correspond to the 2-3 dopaminergic soma between the AOTU and the AL described by Tedjakumala et al. [102]. Because of their localization rather dorso-lateral to the lobula, the MF3 neurons could not be part of the dopaminergic C4 cluster [102].
Mercer et al. [103] also located a catecholamine-labeled neuron cluster dorso-medial to the lobula, as did Nässel et al. [149] for serotonin. The MF4 might belong to the ' $\mathrm{S}_{\mathrm{P}}$-cluster' that projects into the central body and the noduli [51].

We also used AmDop3 to detect the dopaminergic D2-like receptor, previously reported to be expressed in the IKC [150]. There were only individual-DAPI identified-KC nuclei being labeled by both, the AmFoxP and the AmDop 3 probes which supports the suggestion by Suenami et al. [151] that AmFoxP expressing $\mathrm{KC}$ could belong to the lKC. However, mostly, AmDop3 and AmFoxP did not overlap. This is consistent with 
Table 4 List of the 11 AmFoxP expressing neuron populations (clusters or groups) and their co-expressed neurotransmitter(s) (Glut: glutamate, Ach: acetylcholine) ordered from anterior to posterior

\begin{tabular}{|c|c|c|c|c|c|}
\hline$\#$ & $\begin{array}{l}\text { Type of AmFoxP neuron } \\
\text { population }\end{array}$ & Localization & Name & Neurotransmitter & Figures \\
\hline 1 & Group & Around (peri) antennal lobe & $\mathrm{pAL}$ & GABA, Glut, Ach & Figure 3 \\
\hline 2 & Cluster & Anteriodorsal to esophageal hole & adES & Glu & Figure 3 \\
\hline 3 & Group & Around (peri) medulla & pME & GABA, Glut, Ach & Figure 4 \\
\hline 4 & Cluster & Lateral to the lateral horn & ILH & Ach & Figure 5 \\
\hline 5 & Cluster & Anteriolateral to the lateral calyx & alLCA & GABA & Figure 6 \\
\hline 6 & Group & Dorsal to the central complex & $d C X$ & Glu, GABA & Figure 8 \\
\hline 7 & Cluster & Ventral to the medial calyx & vMCA & Ach & Figure 8 \\
\hline 8 & cluster & Posteriolateral to the lateral calyx & plLCA & Ach & Figure 10 \\
\hline 9.1 & Cluster & Medioventral to the lobula, anterior & mvLO-a & Ach & Figure 10 \\
\hline 9.2 & Cluster & Medioventral to the lobula, posterior & mvLO-p & Ach & Figure 10 \\
\hline 10 & Cluster & Middle Kenyon cells & mKC & Ach & Figure 12 \\
\hline 11 & Group & Ventral to the gnathal ganglia & vGNG & GABA, Glut, Ach & Figure 7 \\
\hline
\end{tabular}

the idea that there is a separation between different $\mathrm{KC}$ subgroups, but it is not always sharp.

We did detect monoamines also in the previously described dopaminergic clusters $\mathrm{C} 1, \mathrm{C} 3$ and the S-clusters, which we found to be strongly labeled by AmVMAT, whereas $\mathrm{C} 2$ and $\mathrm{C} 4$ were not labeled. This could be due to differential sensitivity of the markers used. The previous studies detected immunoreactivity for Tyrosine Hydroxylase $(\mathrm{TH})$ the enzyme that catalyzes L-tyrosine to L-DOPA, the precursor for dopamine [51] and immunoreactivity for GABA [102]. Furthermore, our staining labeled the large and strongly expressing octopaminergic VUMmx neurons ventral to the GNG [53]. We unambiguously identified the VUMmx neurons by their projection pattern using neuronal backfilling $[53,83]$. We also observed some very weakly-labeled $A m V M A T$ neurons close to the AL that might express serotonin as described in Dacks et al. [152].

In vertebrates, FoxP2 is expressed in the ventral tegmental area and substantia nigra $[20,23]$ which are the main sources of striatal and limbic forebrain dopamine. However, explicit cellular co-localization of dopamine and FoxP2 has not been reported for these regions.

\section{Neurotransmitter profile of vertebrate FoxP neurons}

Some developmentally relevant transcription factors are only expressed in neurons of a particular neurotransmitter type [153, 154]. This is not the case in our study of honeybees and also not in vertebrates. For instance, FoxP2 and GABA co-occur in the Purkinje cells, striatal medium spiny neurons [14, 20], 'arkypallidal' neurons of the external Globus pallidus [155], neurons ventromedial to the 'Barrington' nucleus ('Bar', pons, brain stem)
[156] and in the parabrachial nucleus ('PB', pons, brain stem) [157]. FoxP2 and glutamate co-occur in pyramidal neurons in the cortex [20], the dLGN (thalamus) [46], neurons dorsolateral to the Bar nucleus [156], in the subparabrachial nucleus [158] and in the PB (pons, brain stem) [157]. Cholinergic spinal motor neurons express FoxP1, FoxP2 and FoxP4 during neuronal differentiation [159-162]. FoxP2-expressing neurons in the substantia nigra [20] are likely to be dopaminergic. Which of these neuron populations might have similarities (beyond FoxP and a specific neurotransmitter profile) to neurons in honeybee, requires more comparative information on molecular profiles of particular AmFoxP expressing neurons, their anatomical connections and their function.

\section{Aging/environmental stimulus manipulation}

Age and environmental factors determine many aspects of life in honeybees. For this reason, we investigated whether age or environmental conditions play a role in the co-expression of neurotransmitters and AmFoxP in the analyzed neuron populations. After emerging from their cells, honeybee workers stay in the hive for about 3 weeks and perform tasks sequentially, i.e. cell cleaning, nursing, wax production and guarding. Subsequently, they start to forage for water, nectar or pollen and communicate their findings with the highly sophisticated 'waggle dance' behavior to their sisters in the hive $[38-41,163]$. They die about 6 weeks after emersion. Honeybee brain anatomy [164] and the expression levels of certain proteins like CREB [165], bruchpilot [166] vitellogenin [167], synapsin [168] or neuropeptides [169] vary with age and experience. Seasonal changes in gene expression were also observed 
[170-172]. FoxP2 levels also change in a specific brain area of songbirds with age [14] and as a result of singing [47-49]. For this reason, we hypothesized that some AmFoxP clusters and groups might be dynamic in terms of AmFoxP and neurotransmitter expression. However, we could identify the clusters and groups based on FoxP expression reliably in all brain samples analyzed, regardless of age and environmental condition. While this finding is not quantitative it suggests that under the housing and seasonal conditions we used AmFoxP and the associated neurotransmitters did not vary noticeably. We conclude that the neuron populations, we identified, are not functionally restricted to certain life-cycle phases, but instead needed for behavior that might be constantly relevant. Using RT-qPCR Kiya et al. [31] detected an age-dependent increase of AmFoxP expression in whole-brain samples from eclosion to age 34 days but no significant differences between nurse and worker bees. We also found an increase of AmFoxP transcript from pupae to workers aged 33 days [25]. We conclude that the results of Kiya et al. [31] and ours reflect a different sensitivity than our in situ hybridization protocol affords. A combination of laser-capture and RT-qPCR could more precisely quantify the actual AmFoxP amounts within the eleven clusters and groups at different life history stages.

\section{Conclusion}

In summary we showed that each of the seven AmFoxP neuron clusters co-expressed only one particular neurotransmitter whereas the four groups co-expressed more than one neurotransmitter and might therefore be further sub-divided. Five clusters expressed AmVAchT and are therefore excitatory whereas one cluster (alLCA) co-expressed $A m G a d$ and is therefore inhibitory. The glutamatergic adES cluster could be inhibitory or excitatory, depending on the receptor type in the postsynapse. None of the eleven AmFoxP neuron populations co-expressed AmVMAT, they are therefore not modulatory. However, a small number of isolated neurons in different parts of the central brain showed co-expression of AmFoxP and AmVMAT.

All eleven AmFoxP neuron populations kept their neurotransmitter profiles across ages and under different conditions of sensory and motor deprivation. This indicates that the FoxP expressing neurons in honeybees do not undergo neurotransmitter switching [173]. Our present data provide a framework to pursue further comparative studies on the function of particular populations of invertebrate and vertebrate FoxP neurons in the context of 'deep homology', which can lead to insights to questions of evolutionary conservation and novelty.

\section{Abbreviations}

See Table 5.

\section{Table 5 List of anatomical and molecular abbreviations}

\begin{tabular}{|c|c|}
\hline \multicolumn{2}{|c|}{ Anatomical abbreviations } \\
\hline $\mathrm{AL}$ & Antennal lobe \\
\hline$C X$ & Central complex \\
\hline $\mathrm{CBU} / \mathrm{CBL}$ & Central body, upper and lower division \\
\hline ES & Esophageal hole \\
\hline GNG & Gnathal ganglia \\
\hline $\mathrm{KC}(\mathrm{sKC}, \mathrm{IKC}, \mathrm{mKC})$ & Small, large and middle Kenyon cells \\
\hline LA & Lamina \\
\hline LCA & Lateral calyx \\
\hline LH & Lateral horn \\
\hline LO & Lobula \\
\hline MCA & Medial calyx \\
\hline ME & Medulla \\
\hline OL & Optic lobe \\
\hline P & Protocerebrum \\
\hline PB & Protocerebral bridge \\
\hline PLP & Posteriolateral protocerebrum \\
\hline$A m$ & Apis mellifera \\
\hline \multicolumn{2}{|c|}{ Molecular abbreviations } \\
\hline AmVAchT & The honeybee vesicular transporter for acetylcholine \\
\hline AmDop3 & The honeybee D2-like dopamine receptor \\
\hline AmVGluT & The honeybee vesicular transporter for glutamate \\
\hline AmGad & The honeybee glutamate decarboxylase \\
\hline AmVIAAT & $\begin{array}{l}\text { The honeybee vesicular transporter of inhibitory } \\
\text { amino acids }\end{array}$ \\
\hline AmVMAT & The honeybee vesicular transporter for monoamines \\
\hline $\mathrm{dlSH}$ & Double-lable in situ hybridization \\
\hline GABA & Gamma-aminobutyric acid \\
\hline glu & Glutamate \\
\hline -ir & Immunoreactive immunoreactive \\
\hline
\end{tabular}




\section{Authors' contributions}

CS, AS and GL designed the study. AS, JA and JM generated dISH data. AS performed confocal imaging. GL designed and performed the RT-qPCR experiments. AS and CS analyzed the data and wrote the manuscript. GL contributed to the analysis and writing. All authors read and approved the final manuscript.

\section{Author details}

1 Department of Animal Behavior, Freie Universität Berlin, Takustraße 6 , 14195 Berlin, Germany. ${ }^{2}$ Department of Neurobiology, Freie Universität Berlin, Königin-Luise-Straße 28-30, 14195 Berlin, Germany.

\section{Acknowledgements}

We thank Peter Knoll for his help with handling the bees, especially for the manipulation experiments. We thank Anja Slowinski for help with generating RT-qPCR data. For providing figure artwork we thank Marco Schubert and for confocal microscope equipment we would like to acknowledge the assistance of the Core Facility BioSupraMol supported by the DFG.

\section{Authors' information}

The present study is part of AS Doctoral project under the supervision of CS, in collaboration with GL. JM did his BA thesis with AS and CS, JA contributed as a student assistant to the laboratory work of GL and AS.

\section{Competing interests}

The authors declare that they have no competing interests.

\section{Availability of data and materials}

The data and materials are available from the corresponding author upon request.

\section{Consent for publication}

Not applicable.

\section{Ethics approval and consent to participate}

Not applicable.

\section{Funding}

AS was funded by a Ph.D. scholarship of the International Research School in Molecular Neurobiology (MolNeuro), part of the Helmholtz foundation. GL and JA were funded by a Research Grant of the DFG: "Molecular correlates of memory phases and development of molecular tools to modify brain physiology in the honeybee" (219171051).

\section{Publisher's Note}

Springer Nature remains neutral with regard to jurisdictional claims in published maps and institutional affiliations.

Received: 31 May 2018 Accepted: 29 October 2018 Published online: 06 November 2018

\section{References}

1. Golson ML, Kaestner $\mathrm{KH}$. Fox transcription factors: from development to disease. Development. 2016;143(24):4558-70.

2. Konopka G, Roberts TF. Insights into the neural and genetic basis of vocal communication. Cell. 2016;164(6):1269-76.

3. Bowers JM, Konopka G. The role of the FOXP family of transcription factors in ASD. Dis Mark. 2012;33(5):251-60.

4. Le Fevre AK, Taylor S, Malek NH, Horn D, Carr CW, Abdul-Rahman OA, et al. FOXP1 mutations cause intellectual disability and a recognizable phenotype. Am J Med Genet Part A. 2013;161(12):3166-75.

5. Reuter MS, Riess A, Moog U, Briggs TA, Chandler KE, Rauch A, et al FOXP2 variants in 14 individuals with developmental speech and language disorders broaden the mutational and clinical spectrum. J Med Genet. 2017;54(1):64-72.

6. Charng WL, Karaca E, Coban Akdemir Z, Gambin T, Atik MM, Gu S, et al. Exome sequencing in mostly consanguineous Arab families with neurologic disease provides a high potential molecular diagnosis rate. BMC Med Genom. 2016;9(1):42.

7. Lai CSL, Fisher SE, Hurst JA, Vargha-Khadem F, Monaco AP. A forkheaddomain gene is mutated in a severe speech and language disorder. Nature. 2001:413(6855):519-23.

8. Schulze K, Vargha-Khadem F, Mishkin M. Phonological working memory and FOXP2. Neuropsychologia. 2018;108:147-52.

9. Gong X, Jia M, Ruan Y, Shuang M, Liu J, Wu S, et al. Association between the FOXP2 gene and autistic disorder in Chinese population. Am J Med Genet Part B Neuropsychiatr Genet. 2004;127B(1):113-6.

10. Chien YL, Wu YY, Chen HI, Tsai WC, Chiu YN, Liu SK, et al. The central nervous system patterning gene variants associated with clinical symptom severity of autism spectrum disorders. J Formos Med Assoc. 2017;116(10):755-64

11. Morgan A, Fisher S, Scheffer I, Hildebrand M. FOXP2-related speech and language disorders. In: Adam MPAH, Pagon RA, et al., editors. June 23 2016 ed. GeneReviews ${ }^{\circledR} ; 2016$.

12. Kaestner $\mathrm{KH}$, Knöchel W, Martínez DE. Unified nomenclature for the winged helix/forkhead transcription factors. Genes Dev. 2000;14(2):142-6.

13. Horng S, Kreiman G, Ellsworth C, Page D, Blank M, Millen K, et al. Differential gene expression in the developing lateral geniculate nucleus and medial geniculate nucleus reveals novel roles for Zic4 and Foxp2 in visual and auditory pathway development. J Neurosci. 2009;29(43):13672-83.

14. Haesler S, Wada K, Nshdejan A, Morrisey EE, Lints T, Jarvis ED, et al. FoxP2 expression in avian vocal learners and non-learners. J Neurosci. 2004; 24(13):3164

15. Wohlgemuth S, Adam I, Scharff C. FoxP2 in songbirds. Curr Opin Neurobiol. 2014;28:86-93.

16. Hannenhalli $\mathrm{S}$, Kaestner $\mathrm{KH}$. The evolution of Fox genes and their role in development and disease. Nat Rev Genet. 2009;10(4):233-40.

17. Takahashi H, Takahashi K, Liu F-C. FOXP genes, neural development, speech and language disorders. In: Maiese K, editor. Forkhead transcription factors: vital elements in biology and medicine. New York: Springer; 2010. p. 117-29.

18. Lai CSL, Gerrelli D, Monaco AP, Fisher SE, Copp AJ. FOXP2 expression during brain development coincides with adult sites of pathology in a severe speech and language disorder. Brain. 2003;126(11):2455-62.

19. Shu W, Yang H, Zhang L, Lu MM, Morrisey EE. Characterization of a new subfamily of winged-helix/forkhead (fox) genes that are expressed in the lung and act as transcriptional repressors. J Biol Chem. 2001;276(29):27488-97.

20. Campbell P, Reep RL, Stoll ML, Ophir AG, Phelps SM. Conservation and diversity of Foxp2 expression in muroid rodents: functional implications. J Comp Neurol. 2009;512(1):84-100.

21. Mendoza E, Tokarev K, Düring DN, Retamosa EC, Weiss M, Arpenik $\mathrm{N}$, et al. Differential coexpression of FoxP1, FoxP2, and FoxP4 in the Zebra Finch (Taeniopygia guttata) song system. J Comp Neurol. 2015;523(9):1318-40

22. Chen Q, Heston JB, Burkett ZD, White SA. Expression analysis of the speech-related genes FoxP1 and FoxP2 and their relation to singing behavior in two songbird species. J Exp Biol. 2013:216(19):3682-92.

23. Ferland RJ, Cherry TJ, Preware PO, Morrisey EE, Walsh CA. Characterization of Foxp2 and Foxp 1 mRNA and protein in the developing and mature brain. J Comp Neurol. 2003;460(2):266-79.

24. Shimeld SM, Degnan B, Luke GN. Evolutionary genomics of the Fox genes: origin of gene families and the ancestry of gene clusters. Genomics. 2010;95(5):256-60.

25. Schatton A, Mendoza E, Grube K, Scharff C. FoxP in bees: a comparative study on the developmental and adult expression pattern in three bee species considering isoforms and circuitry. J Comp Neurol. 2018;526(9):1589-610.

26. Adell T, Muller WE. Isolation and characterization of five Fox (Forkhead) genes from the sponge Suberites domuncula. Gene. 2004:334:35-46.

27. Lawton KJ, Wassmer TL, Deitcher DL. Conserved role of Drosophila melanogaster FoxP in motor coordination and courtship song. Behav Brain Res. 2014:268:213-21. 
28. DasGupta S, Ferreira CH, Miesenböck G. FoxP influences the speed and accuracy of a perceptual decision in Drosophila. Science. 2014;344(6186):901-4.

29. Mendoza E, Colomb J, Rybak J, Pflüger H-J, Zars T, Scharff C, et al. Drosophila FoxP mutants are deficient in operant self-learning. PLoS ONE. 2014;9(6):e100648.

30. Groschner LN, Chan WHL, Bogacz R, DasGupta S, Miesenbock G. Dendritic integration of sensory evidence in perceptual decision-making. Cell. 2018;173(4):894.e13-905.e13.

31. Kiya T, Itoh Y, Kubo T. Expression analysis of the FoxP homologue in the brain of the honeybee Apis mellifera. Insect Mol Biol. 2008;17(1):53-60.

32. Schatton A, Scharff C. FoxP expression identifies a Kenyon cell subtype in the honeybee mushroom bodies linking them to fruit fly a $\beta c$ neurons. Eur J Neurosci. 2017;46(9):2534-41.

33. Santos ME, Athanasiadis A, Leitão AB, DuPasquier L, Sucena É. Alternative splicing and gene duplication in the evolution of the FoxP gene subfamily. Mol Biol Evol. 2011;28(1):237-47.

34. Srinivasan MV. Honey bees as a model for vision, perception, and cognition. Annu Rev Entomol. 2010;55(1):267-84.

35. Menzel R. The honeybee as a model for understanding the basis of cognition. Nat Rev Neurosci. 2012;13(11):758-68.

36. Eisenhardt D. Molecular mechanisms underlying formation of longterm reward memories and extinction memories in the honeybee (Apis mellifera). Learn Memory. 2014:21(10):534-42.

37. Menzel R, Greggers U, Smith A, Berger S, Brandt R, Brunke S, et al. Honey bees navigate according to a map-like spatial memory. Proc Natl Acad Sci USA. 2005;102(8):3040-5.

38. von Frisch K. Decoding the language of the bee. Science. 1974;185:663-8.

39. von Frisch K. Die Tänze der Bienen. Österr Zool Zeit. 1946;1:1-48.

40. Schürch R, Couvillon MJ, Beekman M. Ballroom biology: recent insights into honey bee waggle dance communications. Front Ecol Evol. 2016:3:147.

41. von Frisch K. Über die "Sprache" der Bienen, eine tier-psychologische Untersuchung. Zool Jahrb. 1923;40:1-186.

42. Avargues-Weber A, Giurfa M. Conceptual learning by miniature brains. Proc Biol Sci. 2013;280(1772):20131907.

43. Perry CJ, Barron AB. Honey bees selectively avoid difficult choices. Proc Natl Acad Sci. 2013;110(47):19155-9.

44. Howard SR, Avarguès-Weber A, Garcia JE, Greentree AD, Dyer AG. Numerical ordering of zero in honey bees. Science. 2018:360(6393):1124-6.

45. Duffy KR, Holman KD, Mitchell DE. Shrinkage of X cells in the lateral geniculate nucleus after monocular deprivation revealed by FoxP2 labeling. Vis Neurosci. 2014;31(3):253-61.

46. Iwai L, Ohashi Y, van der List D, Usrey WM, Miyashita Y, Kawasaki H. FoxP2 is a Parvocellular-specific transcription factor in the visual thalamus of monkeys and ferrets. Cereb Cortex (New York, NY). 2013;23(9):2204-12.

47. Miller JE, Spiteri E, Condro MC, Dosumu-Johnson RT, Geschwind DH, White SA. Birdsong decreases protein levels of FoxP2, a molecule required for human speech. J Neurophysiol. 2008;100(4):2015-25.

48. Teramitsu I, White SA. FoxP2 regulation during undirected singing in adult songbirds. J Neurosci. 2006;26(28):7390-4.

49. Adam I, Mendoza E, Kobalz U, Wohlgemuth S, Scharff C. FoxP2 directly regulates the reelin receptor VLDLR developmentally and by singing. Mol Cell Neurosci. 2016;74:96-105.

50. Schäfer S, Bicker G. Distribution of GABA-like immunoreactivity in the brain of the honeybee. J Comp Neurol. 1986;246(3):287-300.

51. Schäfer S, Rehder V. Dopamine-like immunoreactivity in the brain and suboesophageal ganglion of the honeybee. J Comp Neurol. 1989;280(1):43-58.

52. Bicker G, Schafer S, Ottersen O, Storm-Mathisen J. Glutamate-like immunoreactivity in identified neuronal populations of insect nervous systems. J Neurosci. 1988;8(6):2108-22.

53. Kreissl S, Eichmüller S, Bicker G, Rapus J, Eckert M. Octopamine-like immunoreactivity in the brain and subesophageal ganglion of the honeybee. J Comp Neurol. 1994;348(4):583-95.

54. Schürmann F-W, Ottersen OP, Honegger H-W. Glutamate-like immunoreactivity marks compartments of the mushroom bodies in the brain of the cricket. J Comp Neurol. 2000;418(2):227-39.
55. Sinakevitch I, Farris SM, Strausfeld NJ. Taurine-, aspartate- and glutamate-like immunoreactivity identifies chemically distinct subdivisions of Kenyon cells in the cockroach mushroom body. J Comp Neurol. 2001;439(3):352-67.

56. Kreissl S, Bicker G. Histochemistry of acetylcholinesterase and immunocytochemistry of an acetylcholine receptor-like antigen in the brain of the honeybee. J Comp Neurol. 1989;286(1):71-84.

57. Fusca D, Husch A, Baumann A, Kloppenburg P. Choline acetyltransferase-like immunoreactivity in a physiologically distinct subtype of olfactory nonspiking local interneurons in the cockroach (Periplaneta americana). J Comp Neurol. 2013;521(15):3556-69.

58. Kiya T, Kubo T. Analysis of GABAergic and non-GABAergic neuron activity in the optic lobes of the forager and re-orienting worker honeybee (Apis mellifera L.). PLoS ONE. 2010;5(1):e8833.

59. Kucharski R, Ball EE, Hayward DC, Maleszka R. Molecular cloning and expression analysis of a cDNA encoding a glutamate transporter in the honeybee brain. Gene. 2000;242(1-2):399-405.

60. Thany SH, Lenaers G, Crozatier M, Armengaud C, Gauthier M. Identification and localization of the nicotinic acetylcholine receptor alpha3 mRNA in the brain of the honeybee Apis mellifera. Insect Mol Biol. 2003;12(3):255-62.

61. Zannat MT, Locatelli F, Rybak J, Menzel R, Leboulle G. Identification and localisation of the NR1 sub-unit homologue of the NMDA glutamate receptor in the honeybee brain. Neurosci Lett. 2006;398(3):274-9.

62. Sinakevitch I, Mustard JA, Smith BH. Distribution of the octopamine receptor AmOA1 in the honey bee brain. PLoS ONE. 2011;6(1):e14536.

63. El Hassani AK, Schuster S, Dyck Y, Demares F, Leboulle G, Armengaud C. Identification, localization and function of glutamate-gated chloride channel receptors in the honeybee brain. Eur J Neurosci. 2012;36(4):2409-20

64. Démares F, Raymond V, Armengaud C. Expression and localization of glutamate-gated chloride channel variants in honeybee brain (Apis mellifera). Insect Biochem Mol Biol. 2013;43(1):115-24.

65. Humphries MA, Mustard JA, Hunter SJ, Mercer A, Ward V, Ebert PR. Invertebrate D2 type dopamine receptor exhibits age-based plasticity of expression in the mushroom bodies of the honeybee brain. J Neurobiol. 2003;55(3):315-30.

66. Ultsch A, Schuster CM, Laube B, Schloss P, Schmitt B, Betz H. Glutamate receptors of Drosophila melanogaster: cloning of a kainate-selective subunit expressed in the central nervous system. Proc Natl Acad Sci. 1992;89(21):10484-8.

67. Ultsch A, Schuster CM, Laube B, Betz H, Schmitt B. Glutamate receptors of Drosophila melanogaster. FEBS Lett. 1993;324(2):171-7.

68. Romero-Calderón R, Uhlenbrock G, Borycz J, Simon AF, Grygoruk A, Yee $\mathrm{SK}$, et al. A glial variant of the vesicular monoamine transporter is required to store histamine in the drosophila visual system. PLoS Genet. 2008:4(11):e1000245.

69. Homberg U. Neurotransmitters and neuropeptides in the brain of the locust. Microsc Res Tech. 2002;56(3):189-209.

70. Breer H. Neurochemistry of cholinergic synapses in insects. In: von Keyserlingk HC, Jäger A, von Szczepanski C, editors. Approaches to new leads for insecticides. Berlin: Springer; 1985. p. 89-99.

71. Goldberg F, Grünewald B, Rosenboom H, Menzel R. Nicotinic acetylcholine currents of cultured Kenyon cells from the mushroom bodies of the honey bee Apis mellifera. J Physiol. 1999;514(Pt 3):759-68.

72. Barbara GS, Zube C, Rybak J, Gauthier M, Grünewald B. Acetylcholine, GABA and glutamate induce ionic currents in cultured antennal lobe neurons of the honeybee, Apis mellifera. J Comp Physiol A. 2005;191(9):823-36.

73. Müßig L, Richlitzki A, Rößler R, Eisenhardt D, Menzel R, Leboulle $G$. Acute disruption of the NMDA receptor subunit NR1 in the honeybee brain selectively impairs memory formation. J Neurosci. 2010;30(23):7817-25.

74. Démares F, Drouard F, Massou I, Crattelet C, Lœuillet A, Bettiol C, et al. Differential involvement of glutamate-gated chloride channel splice variants in the olfactory memory processes of the honeybee Apis mellifera. Pharmacol Biochem Behav. 2014;124:137-44.

75. Liu WW, Wilson RI. Glutamate is an inhibitory neurotransmitter in the Drosophila olfactory system. Proc Natl Acad Sci USA. 2013;110(25):10294-9. 
76. Enell L, Hamasaka Y, Kolodziejczyk A, Nässel DR. Y-Aminobutyric acid (GABA) signaling components in Drosophila: immunocytochemical localization of GABAB receptors in relation to the GABAA receptor subunit RDL and a vesicular GABA transporter. J Comp Neurol. 2007:505(1):18-31.

77. Sattelle DB, Lummis SCR, Wong JFH, Rauh JJ. Pharmacology of insect GABA receptors. Neurochem Res. 1991;16(3):363-74.

78. El Hassani AK, Giurfa M, Gauthier M, Armengaud C. Inhibitory neurotransmission and olfactory memory in honeybees. Neurobiol Learn Memory. 2008;90(4):589-95.

79. Raccuglia D, Mueller U. Focal uncaging of GABA reveals a temporally defined role for GABAergic inhibition during appetitive associative olfactory conditioning in honeybees. Learn Memory. 2013;20(8):410-6.

80. Greer CL, Grygoruk A, Patton DE, Ley B, Romero-Calderon R, Chang $\mathrm{H}-\mathrm{Y}$, et al. A splice variant of the Drosophila vesicular monoamine transporter contains a conserved trafficking domain and functions in the storage of dopamine, serotonin, and octopamine. J Neurobiol. 2005;64(3):239-58.

81. Waddell S. Reinforcement signalling in Drosophila; dopamine does it all after all. Curr Opin Neurobiol. 2013;23(3):324-9.

82. Kaneko T, Macara AM, Li R, Hu Y, Iwasaki K, Dunnings Z, et al. Serotonergic Modulation enables pathway-specific plasticity in a developing sensory circuit in Drosophila. Neuron. 2017;95(3):623.e4-38.e4.

83. Hammer M. An identified neuron mediates the unconditioned stimulus in associative olfactory learning in honeybees. Nature. 1993;366:59.

84. Kamhi JF, Traniello JFA. Biogenic amines and collective organization in a superorganism: neuromodulation of social behavior in ants. Brain Behav Evol. 2013;82(4):220-36.

85. Schulz DJ, Barron AB, Robinson GE. A Role for octopamine in honey bee division of labor. Brain Behav Evol. 2002;60(6):350-9.

86. Damrau C, Toshima N, Tanimura T, Brembs B, Colomb J. Octopamine and tyramine contribute separately to the counter-regulatory response to sugar deficit in Drosophila. Front Syst Neurosci. 2017;11:100.

87. Stevenson PA, Dyakonova V, Rillich J, Schildberger K. Octopamine and experience-dependent modulation of aggression in crickets. J Neurosci. 2005;25(6):1431-41.

88. Nall AH, Sehgal A. Small-molecule screen in adult Drosophila identifies VMAT as a regulator of sleep. J Neurosci. 2013;33(19):8534-40.

89. Katz P, Grillner S, Wilson R, Borst A, Greenspan R, Buzsáki G, et al. Vertebrate versus invertebrate neural circuits. Curr Biol. 2013;23(12):R504-6.

90. Shubin N, Tabin C, Carroll S. Deep homology and the origins of evolutionary novelty. Nature. 2009:457:818.

91. Strausfeld NJ, Hirth F. Deep homology of arthropod central complex and vertebrate basal ganglia. Science. 2013;340(6129):157-61.

92. Altschul SF, Madden TL, Schäffer AA, Zhang J, Zhang Z, Miller W, et al. Gapped BLAST and PSI-BLAST: a new generation of protein database search programs. Nucleic Acids Res. 1997;25(17):3389-402.

93. Koressaar T, Remm M. Enhancements and modifications of primer design program Primer3. Bioinformatics. 2007;23(10):1289-91.

94. Strutz A, Soelter J, Baschwitz A, Farhan A, Grabe V, Rybak J, et al. Decoding odor quality and intensity in the Drosophila brain. ELife. 2014;3:e04147.

95. Bicker G, Schäfer S, Kingan TG. Mushroom body feedback interneurones in the honeybee show GABA-like immunoreactivity. Brain Res. 1985;360(1-2):394-7.

96. Rybak J, Menzel R. Anatomy of the mushroom bodies in the honey bee brain: the neuronal connections of the alpha-lobe. J Comp Neurol. 1993;334(3):444-65.

97. Bicker G. Histochemistry of classical neurotransmitters in antennal lobes and mushroom bodies of the honeybee. Microsc Res Tech. 1999;45(3):174-83.

98. Warren B, Kloppenburg P. Rapid and Slow chemical synaptic interactions of cholinergic projection neurons and GABAergic local interneurons in the insect antennal lobe. J Neurosci. 2014;34(39):13039-46.

99. Ito K, Shinomiya K, Ito M, Armstrong JD, Boyan G, Hartenstein $\checkmark$, et al. A systematic nomenclature for the insect brain. Neuron. 2014;81(4):755-65.

100. Brandt R, Rohlfing T, Rybak J, Krofczik S, Maye A, Westerhoff M, et al. Three-dimensional average-shape atlas of the honeybee brain and its applications. J Comp Neurol. 2005;492(1):1-19.
101. Lai S-L, Awasaki T, Ito K, Lee T. Clonal analysis of Drosophila antennal lobe neurons: diverse neuronal architectures in the lateral neuroblast lineage. Development. 2008;135(17):2883-93.

102. Tedjakumala SR, Rouquette J, Boizeau ML, Mesce KA, Hotier L, Massou I, et al. A tyrosine-hydroxylase characterization of dopaminergic neurons in the honey bee brain. Front Syst Neurosci. 2017;11(47):47.

103. Mercer AR, Mobbs PG, Davenport AP, Evans PD. Biogenic amines in the brain of the honeybee Apis mellifera. Cell Tissue Res. 1983;234(3):655-77.

104. Graham SA, Fisher SE. Understanding language from a genomic perspective. Annu Rev Genet. 2015;49(1):131-60.

105. Haesler S, Rochefort C, Georgi B, Licznerski P, Osten P, Scharff C. Incomplete and inaccurate vocal imitation after knockdown of FoxP2 in Songbird basal ganglia nucleus area X. PLoS Biol. 2007;5(12):e321.

106. Schreiweis $C$, Bornschein U, Burguière E, Kerimoglu C, Schreiter S, Dannemann $M$, et al. Humanized Foxp2 accelerates learning by enhancing transitions from declarative to procedural performance. Proc Natl Acad Sci. 2014;111(39):14253-8.

107. Gaub S, Fisher SE, Ehret G. Ultrasonic vocalizations of adult male Foxp2mutant mice: behavioral contexts of arousal and emotion. Genes Brain Behav. 2016;15(2):243-59.

108. Chabout J, Sarkar A, Patel SR, Radden T, Dunson DB, Fisher SE, et al. A Foxp2 mutation implicated in human speech deficits alters sequencing of ultrasonic vocalizations in adult male mice. Front Behav Neurosci. 2016;10:197.

109. Heston JB, White SA. Behavior-linked FoxP2 regulation enables zebra finch vocal learning. J Neurosci. 2015;35(7):2885-94.

110. Kurt S, Fisher SE, Ehret G. Foxp2 mutations impair auditory-motor association learning. PLoS ONE. 2012;7(3):e33130.

111. Lee HH, Frasch M. Survey of forkhead domain encoding genes in the Drosophila genome: classification and embryonic expression patterns. Dev Dyn. 2004;229(2):357-66.

112. Song $X$, Tang $Y$, Wang $Y$. Genesis of the vertebrate FoxP subfamily member genes occurred during two ancestral whole genome duplication events. Gene. 2016;588(2):156-62.

113. Wang VY, Hassan BA, Bellen HJ, Zoghbi HY. Drosophila atonal fully rescues the phenotype of math1 null mice: new functions evolve in new cellular contexts. Curr Biol. 2002;12(18):1611-6.

114. Quiring R, Walldorf U, Kloter U, Gehring W. Homology of the eyeless gene of Drosophila to the small eye gene in mice and Aniridia in humans. Science. 1994;265(5173):785-9.

115. Williams MJ, Goergen P, Rajendran J, Zheleznyakova G, Hägglund MG, Perland E, et al. Obesity-linked homologues TfAP-2 and Twz establish meal frequency in Drosophila melanogaster. PLoS Genet. 2014;10(9):e1004499.

116. Kalousova A, Mavropoulos A, Adams BA, Nekrep N, Li Z, Krauss S, et al. Dachshund homologues play a conserved role in islet cell development. Dev Biol. 2010;348(2):143-52.

117. Villar D, Flicek P, Odom DT. Evolution of transcription factor binding in metazoans-mechanisms and functional implications. Nat Rev Genet. 2014;15:221.

118. Liang ZS, Nguyen T, Mattila HR, Rodriguez-Zas SL, Seeley TD, Robinson GE. Molecular determinants of scouting behavior in honey bees. Science 2012:335(6073):1225-8.

119. Dupuis J, Louis T, Gauthier M, Raymond V. Insights from honeybee (Apis mellifera) and fly (Drosophila melanogaster) nicotinic acetylcholine receptors: from genes to behavioral functions. Neurosci Biobehav Rev. 2012;36(6):1553-64.

120. Liebeskind BJ, Hillis DM, Zakon HH, Hofmann HA. Complex homology and the evolution of nervous systems. Trends Ecol Evol. 2016:31(2):127-35.

121. Kloepper TH, Kienle CN, Fasshauer D. An elaborate classification of SNARE proteins sheds light on the conservation of the eukaryotic endomembrane system. Mol Biol Cell. 2007;18(9):3463-71.

122. Ai H, Kai K, Kumaraswamy A, Ikeno H, Wachtler T. Interneurons in the honeybee primary auditory center responding to waggle dance-like vibration pulses. J Neurosci. 2017;37(44):10624-35.

123. Pollack GS. Analysis of temporal patterns of communication signals. Curr Opin Neurobiol. 2001;11(6):734-8.

124. Alluri RK, Rose GJ, Hanson JL, Leary CJ, Vasquez-Opazo GA, Graham JA, et al. Phasic, suprathreshold excitation and sustained inhibition underlie 
neuronal selectivity for short-duration sounds. Proc Natl Acad Sci USA. 2016;113(13):E1927-35.

125. Pfeiffer K, Homberg U. Organization and functional roles of the central complex in the insect brain. Annu Rev Entomol. 2014;59(1):165-84.

126. Stone T, Webb B, Adden A, Weddig NB, Honkanen A, Templin R, et al. An anatomically constrained model for path integration in the bee brain. Curr Biol. 2017;27(20):3069.e11-85.e11.

127. Blenau W, Schmidt M, Faensen D, Schürmann F-W. Neurons with dopamine-like immunoreactivity target mushroom body Kenyon cell somata in the brain of some hymenopteran insects. Int J Insect Morphol Embryol. 1999;28(3):203-10.

128. Shapira M, Thompson CK, Soreq H, Robinson GE. Changes in neuronal acetylcholinesterase gene expression and division of labor in honey bee colonies. J Mol Neurosci. 2001;17(1):1-12.

129. De La Porte S, Vallette FM, Grassi J, Vigny M, Koenig J. Presynaptic or postsynaptic origin of acetylcholinesterase at neuromuscular junctions? An immunological study in heterologous nerve-muscle cultures. Dev Biol. 1986;116(1):69-77.

130. Zoli M. Distribution of cholinergic neurons in the mammalian brain with special reference to their relationship with neuronal nicotinic acetylcholine receptors. In: Clementi F, Fornasari D, Gotti C, editors. Neuronal nicotinic receptors. Berlin: Springer; 2000. p. 13-30.

131. Kim YH, Kim JH, Kim K, Lee SH. Expression of acetylcholinesterase 1 is associated with brood rearing status in the honey bee Apis mellifera. Sci Rep. 2017;7:39864.

132. Zimmermann M. Neuronal AChE splice variants and their non-hydrolytic functions: redefining a target of AChE inhibitors? Br J Pharmacol. 2013;170(5):953-67.

133. Barnstedt O, Owald D, Felsenberg J, Brain R, Moszynski J-P, Talbot Clifford B, et al. Memory-relevant mushroom body output synapses are cholinergic. Neuron. 2016;89(6):1237-47.

134. Kahsai L, Carlsson MA, Winther ÅME, Nässel DR. Distribution of metabotropic receptors of serotonin, dopamine, GABA, glutamate, and short neuropeptide F in the central complex of Drosophila. Neuroscience. 2012;208:11-26.

135. Hsu CT, Bhandawat V. Organization of descending neurons in Drosophila melanogaster. Sci Rep. 2016;6:20259.

136. Hasegawa E, Truman JW, Nose A. Identification of excitatory premotor interneurons which regulate local muscle contraction during Drosophila larval locomotion. Sci Rep. 2016;6:30806.

137. Whitfield CW, Cziko A-M, Robinson GE. Gene expression profiles in the brain predict behavior in individual honey bees. Science. 2003;302(5643):296-9.

138. Kaoru T, Fu-Chin L, Katsuiku H, Hiroshi T. Expression of Foxp4 in the developing and adult rat forebrain. J Neurosci Res. 2008;86(14):3106-16.

139. Teramitsu I, Kudo LC, London SE, Geschwind DH, White SA. Parallel oxP1 and FoxP2 expression in songbird and human brain predicts functional interaction. J Neurosci. 2004;24(13):3152-63.

140. Kaoru T, Fu-Chin L, Katsuiku H, Hiroshi T. Expression of Foxp2, a gene involved in speech and language, in the developing and adult striatum. J Neurosci Res. 2003;73(1):61-72.

141. Graybiel AM, Aosaki T, Flaherty AW, Kimura M. The basal ganglia and adaptive motor control. Science. 1994;265(5180):1826.

142. DeLong MR, Georgopoulos AP. Motor functions of the basal ganglia. London: Wiley; 2011.

143. Tewari A, Jog R, Jog MS. The striatum and subthalamic nucleus as independent and collaborative structures in motor control. Front Syst Neurosci. 2016;10:17.

144. Tecuapetla F, Jin X, Lima SQ, Costa RM. Complementary contributions of striatal projection pathways to action initiation and execution. Cell. 2016;166(3):703-15

145. Varga AG, Kathman ND, Martin JP, Guo P, Ritzmann RE. Spatial navigation and the central complex: sensory acquisition, orientation, and motor control. Front Behav Neurosci. 2017;11:4

146. Martin JP, Guo P, Mu L, Harley CM, Ritzmann RE. Central-complex control of movement in the freely walking cockroach. Curr Biol. 2015;25(21):2795-803

147. Strauss R. The central complex and the genetic dissection of locomotor behaviour. Curr Opin Neurobiol. 2002;12(6):633-8.
148. Christensen TA, Waldrop BR, Harrow ID, Hildebrand JG. Local interneurons and information processing in the olfactory glomeruli of the moth Manduca sexta. J Comp Physiol A. 1993;173(4):385-99.

149. Nässel DR. Histamine in the brain of insects: a review. Microsc Res Tech. 1999:44(2-3):121-36.

150. McQuillan HJ, Nakagawa S, Mercer AR. Mushroom bodies of the honeybee brain show cell population-specific plasticity in expression of amine-receptor genes. Learn Memory. 2012;19(4):151-8.

151. Suenami S, Oya S, Kohno H, Kubo T. Kenyon cell subtypes/populations in the honeybee mushroom bodies: possible function based on their gene expression profiles, differentiation, possible evolution, and application of genome editing. Front Psychol. 2018;9:1717.

152. Dacks AM, Reisenman CE, Paulk AC, Nighorn AJ. Histamine-immunoreactive local neurons in the antennal lobes of the hymenoptera. J Comp Neurol. 2010;518(15):2917-33.

153. Juárez-Morales JL, Schulte CJ, Pezoa SA, Vallejo GK, Hilinski WC, England SJ, et al. Evx1 and Evx2 specify excitatory neurotransmitter fates and suppress inhibitory fates through a Pax2-independent mechanism. Neural Dev. 2016;11(1):5.

154. Borromeo MD, Meredith DM, Castro DS, Chang JC, Tung K-C, Guillemot $F$, et al. A transcription factor network specifying inhibitory versus excitatory neurons in the dorsal spinal cord. Development. 2014;141(14):2803-12.

155. Abdi A, Mallet N, Mohamed FY, Sharott A, Dodson PD, Nakamura KC, et al. Prototypic and arkypallidal neurons in the dopamine-intact external globus pallidus. J Neurosci. 2015;35(17):6667-88.

156. Verstegen AMJ, Vanderhorst V, Gray PA, Zeidel ML, Geerling JC. Barrington's nucleus: neuroanatomic landscape of the mouse "pontine micturition center". J Comp Neurol. 2017;525(10):2287-309.

157. Geerling JC, Kim M, Mahoney CE, Abbott SBG, Agostinelli L, Garfield $A S$, et al. Genetic identity of thermosensory relay neurons in the lateral parabrachial nucleus. Am J Physiol Regul Integr Comp Physiol. 2016;310(1):R41-54.

158. Geerling JC, Yokota S, Rukhadze I, Roe D, Chamberlin NL. Kölliker-Fuse GABAergic and glutamatergic neurons project to distinct targets. J Comp Neurol. 2017;525(8):1844-60.

159. Rousso David L, Pearson Caroline A, Gaber ZB, Miquelajauregui A, Li $\mathrm{S}$, Portera-Cailliau $\mathrm{C}$, et al. Foxp-mediated suppression of $\mathrm{N}$-cadherin regulates neuroepithelial character and progenitor maintenance in the CNS. Neuron. 2012:74(2):314-30.

160. Rousso DL, Gaber ZB, Wellik D, Morrisey EE, Novitch BG. Coordinated actions of the Forkhead protein Foxp1 and hox proteins in the columnar organization of spinal motor neurons. Neuron. 2008;59(2):226-40.

161. Dasen JS, De Camilli A, Wang B, Tucker PW, Jessell TM. Hox repertoires for motor neuron diversity and connectivity gated by a single accessory factor, FoxP1. Cell. 2008:134(2):304-16.

162. Morikawa Y, Komori T, Hisaoka T, Senba E. Detailed expression pattern of Foxp 1 and Its possible roles in neurons of the spinal cord during embryogenesis. Dev Neurosci. 2009;31(6):511-22.

163. Seeley TD. Honeybee democracy. Princeton: Princeton University Press; 2010

164. Groh C, Lu Z, Meinertzhagen IA, Rössler W. Age-related plasticity in the synaptic ultrastructure of neurons in the mushroom body calyx of the adult honeybee Apis mellifera. J Comp Neurol. 2012;520(15):3509-27.

165. Gehring KB, Heufelder K, Kersting I, Eisenhardt D. Abundance of phosphorylated Apis mellifera CREB in the honeybee's mushroom body inner compact cells varies with age. J Comp Neurol. 2016;524(6):1165-80.

166. Gehring KB, Heufelder K, Depner H, Kersting I, Sigrist SJ, Eisenhardt D. Age-associated increase of the active zone protein Bruchpilot within the honeybee mushroom body. PLoS ONE. 2017;12(4):e0175894.

167. Amdam GV, Omholt SW. The regulatory anatomy of honeybee lifespan. J Theor Biol. 2002;216(2):209-28

168. Fahrbach SE, Van Nest BN. Synapsin-based approaches to brain plasticity in adult social insects. Curr Opin Insect Sci. 2016;18(Supplement C):27-34.

169. Han B, Fang Y, Feng M, Hu H, Qi Y, Huo X, et al. Quantitative Neuropeptidome analysis reveals neuropeptides are correlated with social behavior regulation of the honeybee workers. J Proteome Res. 2015;14(10):4382-93. 
170. Steinmann N, Corona M, Neumann P, Dainat B. Overwintering is associated with reduced expression of immune genes and higher susceptibility to virus infection in honey bees. PLoS ONE. 2015;10(6):e0129956.

171. Bonnafé E, Alayrangues J, Hotier L, Massou I, Renom A, Souesme G, et al. Monoterpenoid-based preparations in beehives affect learning, memory, and gene expression in the bee brain. Environ Toxicol Chem. 2017;36(2):337-45.
172. Cardoso-Júnior CAM, Eyer M, Dainat B, Hartfelder K, Dietemann V. Social context influences the expression of DNA methyltransferase genes in the honeybee. Sci Rep. 2018;8(1):11076.

173. Spitzer NC. Neurotransmitter switching in the developing and adult brain. Annu Rev Neurosci. 2017:40(1):1-19.
Ready to submit your research? Choose BMC and benefit from:

- fast, convenient online submission

- thorough peer review by experienced researchers in your field

- rapid publication on acceptance

- support for research data, including large and complex data types

- gold Open Access which fosters wider collaboration and increased citations

- maximum visibility for your research: over $100 \mathrm{M}$ website views per year

At BMC, research is always in progress.

Learn more biomedcentral.com/submissions 